\title{
Student Portfolios and the College Admissions Problem
}

\section{Citation}

Chade, H., G. Lewis, and L. Smith. 2014. “Student Portfolios and the College Admissions Problem." The Review of Economic Studies (February 17).

\section{Published Version}

doi:10.1093/restud/rdu003

\section{Permanent link}

http://nrs.harvard.edu/urn-3:HUL.InstRepos:12363836

\section{Terms of Use}

This article was downloaded from Harvard University's DASH repository, and is made available under the terms and conditions applicable to Open Access Policy Articles, as set forth at http:// nrs.harvard.edu/urn-3:HUL.InstRepos:dash.current.terms-of-use\#OAP

\section{Share Your Story}

The Harvard community has made this article openly available.

Please share how this access benefits you. Submit a story.

\section{Accessibility}




\title{
Student Portfolios and the College Admissions Problem*
}

\author{
Hector Chade ${ }^{\dagger} \quad$ Gregory Lewis ${ }^{\ddagger} \quad$ Lones Smith ${ }^{\S}$
}

\author{
First version: July 2003 \\ This version: January 24, 2014
}

\begin{abstract}
We develop a decentralized Bayesian model of college admissions with two ranked colleges, heterogeneous students and two realistic match frictions: students find it costly to apply to college, and college evaluations of their applications are uncertain. Students thus face a portfolio choice problem in their application decision, while colleges choose admissions standards that act like market-clearing prices. Enrollment at each college is affected by the standards at the other college through student portfolio reallocation. In equilibrium, student-college sorting may fail: weaker students sometimes apply more aggressively, and the weaker college might impose higher standards. Applying our framework, we analyze affirmative action, showing how it induces minority applicants to construct their application portfolios as if they were majority students of higher caliber.
\end{abstract}

*Earlier versions were called "The College Admissions Problem with Uncertainty" and "A Supply and Demand Model of the College Admissions Problem". We would like to thank Philipp Kircher (CoEditor) and three anonymous referees for their helpful comments and suggestions. Greg Lewis and Lones Smith are grateful for the financial support of the National Science Foundation. We have benefited from seminars at BU, UCLA, Georgetown, HBS, the 2006 Two-Sided Matching Conference (Bonn), 2006 SED (Vancouver), 2006 Latin American Econometric Society Meetings (Mexico City), and 2007 American Econometric Society Meetings (New Orleans), Iowa State, Harvard/MIT, the 2009 Atlanta NBER Conference, and Concordia. Parag Pathak and Philipp Kircher provided useful discussions of our paper. We are also grateful to John Bound and Brad Hershbein for providing us with student college applications data.

${ }^{\dagger}$ Arizona State University, Department of Economics, Tempe, AZ 85287.

${ }_{\ddagger}^{\ddagger}$ Harvard University, Department of Economics, Cambridge, MA 02138.

${ }^{\S}$ University of Wisconsin, Department of Economics, Madison, WI 53706. 


\section{Introduction}

The college admissions process has lately been the object of much scrutiny, both from academics and in the popular press. This interest owes in part to the competitive nature of college admissions. Schools set admissions standards to attract the best students, and students in turn respond most judiciously in making their application decisions. This paper examines the joint behavior of students and colleges in equilibrium.

We introduce an equilibrium model of college admissions that analyzes the impact of two previously unexplored frictions in the application process: students find it costly to apply to college, and college evaluations of their applications is uncertain. As evidence of the noise in the process, observe that admissions rates are well below $50 \%$ at the most selective colleges, and below $75 \%$ at the median 4 year college 1 This uncertainty prevails conditional on the SAT. Even applicants with perfect SAT scores have no better than a 50\% chance of getting into schools like Harvard, MIT and Princeton Avery, Glickman, Hoxby, and Metrick, 2004). So it is not surprising that most applicants construct thoughtful portfolios that include both "safety" and "stretch" schools.

Despite this uncertainty, the costly nature of applications substantially limits the number of applications sent - three for the median student ${ }^{2}$ A fast growing empirical literature confirms the impact of this friction: for instance, Pallais (2009) finds that when the ACT allowed students to send an extra free application, 20\% of ACT testtakers took advantage of this option. ${ }^{3}$ Small costs can have large effects on application behavior because the marginal benefit of applying falls geometrically in the number of applications. A student applying to identical four-year colleges with a typical $75 \%$ acceptance rate sees the marginal benefit to her 5 th application scaled by $4^{-4}=1 / 256$ : Even if attending college this year is worth $\$ 20000$, the marginal benefit is only $\$ 59$.

Figure 1 illustrates some motivating patterns in the application data. The left panel demonstrates the importance of the application frictions - for the chance of matriculating at a private elite school is significantly higher for students who apply widely $4^{4}$ The right panel plots the average number of applications as a function of the SAT score,

\footnotetext{
${ }^{1}$ Source: Table 329, Digest of Education Statistics, National Center for Education Statistics.

${ }^{2}$ Source: Higher Education Research Institute (HERI), using a large nationally representative survey of college freshman since 1966 (data also used to construct Figure 1; see online appendix for details).

$3_{\text {Steinberg }}(2010)$ reported that colleges who waived application fees saw applications skyrocket.

4 Avery and Kane (2004) study a Boston program giving low-income students advice on how/where to apply; these students matriculated at a higher rate than comparable students elsewhere. Similar results have later been found in field experiments where students received application help: see Bettinger, Long, Oreopoulos, and Sanbonmatsu (2009) and Carrell and Sacerdote (2012).
} 

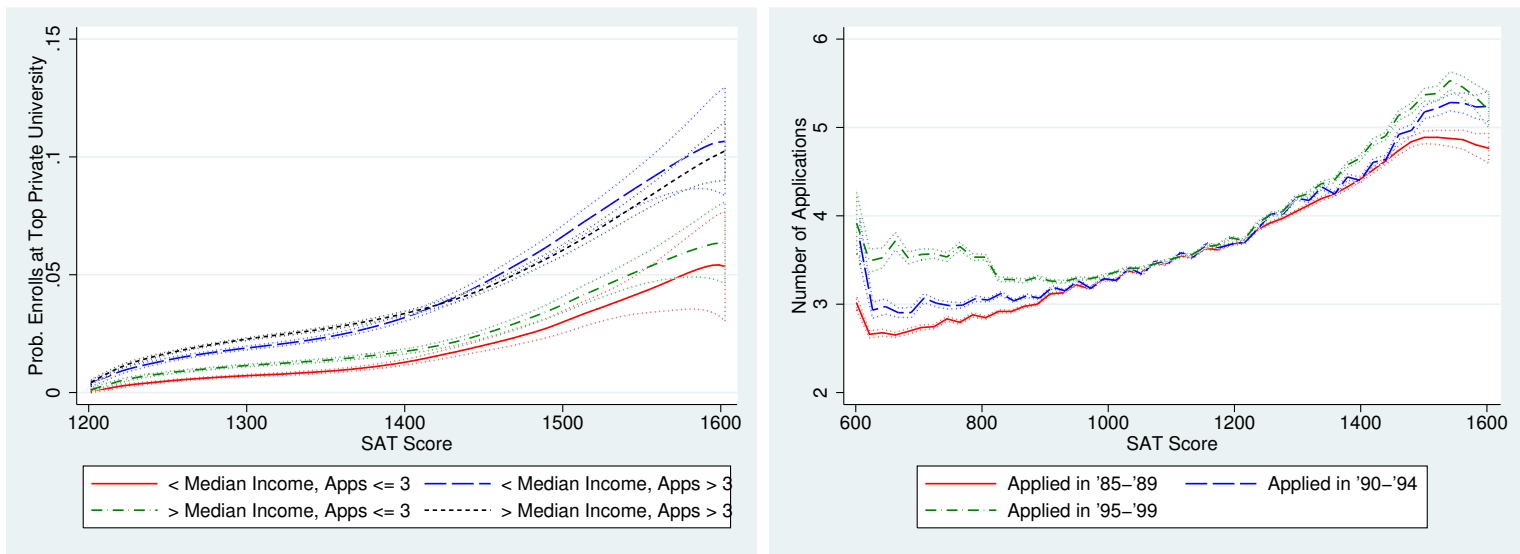

Figure 1: Applications and Matriculation by SAT. The left panel shows the chance of matriculating at a top private university, by number of applications and household income. The right panel shows the estimated relationship between SAT score and number of applications. Estimation is by local linear regression; 95\% confidence intervals are shown as dotted lines.

small but larger than one consistent with our two frictions.$^{5}$

Any model that focuses on these two frictions - costly portfolio choices with incomplete information - must diverge from the approach of the centralized college matching literature (Gale and Shapley, 1962), for it expressly sidesteps such matching frictions. Rather, we analyze an entirely decentralized model that parallels the actual process. It affords sharp conclusions about two key decision margins: how colleges set admission standards and how students formulate their application portfolios.

We assume a heterogeneous population of students, and two ranked colleges - one better and one worse, respectively, called 1 and 2. Like the decentralized model of Avery and Levin (2010), there is a continuum of students; this avoids colleges facing aggregate uncertainty — otherwise, wait-listing is needed, for instance ${ }^{6}$ Colleges seek to fill their capacity with the best students possible, but student calibers are only imperfectly observed. The tandem of costly applications and yet noisy evaluations feeds the intriguing conflict at the heart of the student choice problem: shoot for the Ivy League, settle for the local state school, or apply to both. As we shall see, our paper formalizes the critical roles played by stretch and safety schools. Meanwhile, college enrollments are

\footnotetext{
${ }^{5}$ Admissions rates fall rapidly as college rank rises. So larger portfolios are particularly valuable for high SAT students applying to top-tier colleges, consistent with the right panel of Figure 1.

${ }^{6}$ See Che and Koh (2013) for a college admissions model with aggregate uncertainty. We also omit many real-world elements like financial aid and peer effects, but these are typically ruled out in centralized matching models too. In a recent paper, Azevedo and Leshno (2012) assume a continuum of students in a centralized paper in the spirit of Gale and Shapley (1962), and find that it affords a characterization of equilibrium in terms of supply and demand - one of our observations too.
} 
interdependent, since the students' portfolios depend on the joint college admissions standards, and students accepted at the better college will not attend the lesser one. This asymmetric interdependence leads to many surprising results.

Central to our paper is a theorem characterizing how student acceptance chances at the colleges covary with student caliber. We deduce that as a student's caliber rises, the ratio of his admission chances at college 1 to college 2 rises monotonically. We are thus able to solve the equilibrium in three stages, first deducing how acceptance chances translate into application portfolios, and then seeing how portfolio choices across student calibers relate, for any pair of college admission standards; finally, we compute the derived demand for college slots. We analyze the equilibrium of the induced admissions standards game among colleges through the lens of supply and demand: When a college raises its standards, its enrollment falls both because fewer students make the cut the standards effect - and fewer will apply ex ante — the portfolio effect. Treating admissions standards as prices, these effects reinforce each other. In equilibrium, we uncover a "law of demand", in which a college's enrollment falls in its standard. The portfolio effect increases the elasticity of this demand curve.

Analogous to Bertrand competition with differentiated products, colleges will choose admissions standards to fill their desired enrollment, taking rival standards and the student portfolios as given. An equilibrium occurs when both markets clear and students behave optimally. The model frictions yield some novel comparative statics. For instance, the admissions standards at both colleges fall if college 2 raises its capacity, while lower application costs at either school increase the admissions standards at the better college. We will argue that our equilibrium framework rationalizes the pattern of changing college standards and admission rates recently documented by Hoxby (2009).

In a major thrust of the paper, we ask whether sorting occurs in equilibrium: First, do the better students apply more "aggressively"? Precisely, the best students apply just to college 1; weaker students insure by applying to both colleges; even weaker ones apply just to college 2; and finally, the weakest apply nowhere. Such an application pattern rationalizes the general rise and fall that we observe in the right panel of Figure 1 . Second, does the better college impose higher admissions standards? The answer to this question is no when the lesser college is sufficiently small, for by our "law of demand", college 2 continues to raise its standards as its capacity falls. Failures of student sorting are more subtle: The willingness of students either to $(i)$ gamble on a stretch school or $(i i)$ insure themselves with a safety school may not be monotone in their types. Conversely, 
all equilibria entail sorting when the colleges differ sufficiently in quality and the lower ranked school is not too small. All told, sorting proves elusive with frictions. 7 The college sorting failures that we identify have problematic implications for rankings based on the characteristics of matriculants, such as their SAT scores: Colleges that substantially increase their capacity are penalized, since they must lower their admission standards.

This paper takes very seriously the uncertainty that clouds the student admission process. Students apply to colleges, perhaps knowing their types, or perhaps ignorant of them. Equally well, colleges evaluate students trying to gauge the future stars, and often do not succeed. The best framework for analyzing this world therefore involves two-sided incomplete information. In fact, we later formulate such a richer Bayesian model, and argue that its predictions are well-approximated by ours where students know their types, and colleges observe noisy signals. The sorting failures we claim, as well as the positive theory of how students and colleges react, are in fact robust findings.

We conclude the paper with a topical foray into "affirmative action" for in-state applicants, or other preferred applicant groups. We show that colleges impose different admissions standards so as to equate the "shadow values" of applicants from different groups - a form of third degree price discrimination. This, in turn, affects how students behave: in a simple case, lower caliber applicants of a favored group behave as if they were higher caliber applicants from a non-favored group. This is consistent with the reduction in less-qualified minority applications to selective public schools after the end of affirmative action in California and Texas, documented in Card and Krueger (2005).

\section{The Model}

A. An Overview. The paper introduces three key features - heterogeneous students, portfolio choices with unit application costs, and noisy evaluations by colleges. We impose little additional structure. For instance, we ignore the important and realistic consideration of heterogeneous student preferences over colleges, as well as peer effects.

A central feature of our analysis is modeling college portfolio applications. Student choice is trivial if it is costless, and in practice, such costs can be quite high. Indeed, the

\footnotetext{
${ }^{7}$ This adds to the literature on decentralized frictional matching - e.g., Shimer and Smith (2000), Smith (2006), Chade (2006), and Anderson and Smith (2010). The student portfolio problem in the model is a special case of Chade and Smith (2006). In this sense, our paper also contributes to the directed search literature. See Burdett and Judd (1983), Burdett, Shi, and Wright (2001), Albrecht, Gautier, and Vroman (2003) and Kircher and Galenianos (2006)).
} 
sole purpose of the Common Application is to lower the cost of multiple applications 8

Next, we assume noisy signals of student calibers. This informational friction creates uncertainty for students, and a Bayesian filtering problem for colleges. It captures the difficulty faced by market participants, with students choosing "safety schools" and "stretch schools", and colleges trying to infer the best students from noisy signals. Without noise, sorting would be trivial: Better students would apply and be admitted to better colleges, for their caliber would be correctly inferred and they would be accepted.

We also make two other key modeling choices. First, we assume just two colleges, for the sake of tractability. But as we argue in the conclusion, this is the most parsimonious framework that captures all of our key findings. We also fix the capacity of the two colleges. This is defensible in the short run, and so it is best to interpret our model as focusing on the "short run" analysis of college admissions. We explore the simultaneous game in which students apply to college, and colleges decide whom to admit $?^{9}$

In the interest of tractability, our analysis assumes that the colleges' evaluations of students are conditionally independent. This captures the case where students are apprised of all variables (such as the ACT/SAT or their GPA) common to their applications before applying to college. Students are uncertain as to how these idiosyncratic elements such as college-specific essays and interviews will be evaluated, but believe that the resulting signals are conditionally independent across colleges. We revisit this restriction in Section 6, and argue that our main results on sorting are robust, and that we have analyzed a representative case.

B. The Model. There are two colleges 1 and 2 with capacities $\kappa_{1}$ and $\kappa_{2}$, and a unit mass of students with calibers $x$ whose distribution has a positive density $f(x)$ over $[0, \infty)$. Non-triviality demands that college capacity be insufficient for all students, as $\kappa_{1}+\kappa_{2}<1$. To avoid many subscripts, we shall almost always assume that students pay a separate but common application cost $c>0$ for the two colleges. All students prefer college 1. Everyone receives payoff $v>0$ for attending college $1, u \in(0, v)$ for college 2 , and zero payoff for not attending college. Students maximize expected college

\footnotetext{
${ }^{8}$ This general application form is used by almost 400 colleges, and simplifies college applications. It eliminates idiosyncratic college requirements, but retains separate college application fees.

${ }^{9}$ Epple, Romano, and Sieg (2006) analyze an equilibrium model that includes tuition as a choice variable, price discrimination, peer effects, and students that differ in ability and income. Under single crossing conditions, they obtain positive sorting on ability for each income level. Their model, however, does not include costly applications or noise, thus precluding the portfolio effects we focus on and their implications for sorting. While we do not allow colleges to choose their tuition levels, we do not ignore the role of tuition, for one can simply interpret the benefits of attending college as net benefits.
} 
payoff less application costs. Colleges maximize the total caliber of their student bodies subject to capacity constraints.

Students know their caliber, and colleges do not. Colleges 1 and 2 each just observes a noisy conditionally independent signal of each applicant's caliber. In particular, they do not know where else students have applied. Signals $\sigma$ are drawn from a conditional density function $g(\sigma \mid x)$ on a subinterval of $\mathbb{R}$, with $\operatorname{cdf} G(\sigma \mid x)$. We assume that $g(\sigma \mid x)$ is continuous and obeys the strict monotone likelihood ratio property (MLRP). So $g(\tau \mid x) / g(\sigma \mid x)$ is increasing in the student's type $x$ for all signals $\tau>\sigma$.

Students apply simultaneously to either, both, or neither college, choosing for each caliber $x$, a college application menu $S(x)$ in $\{\varnothing,\{1\},\{2\},\{1,2\}\}$. Colleges choose the set of acceptable student signals. They intuitively should use admission standards to maximize their objective functions, so that college $i$ admits students above a threshold signal $\underline{\sigma}_{i}$. Appendix A.1 proves this given the MLRP property - despite an acceptance curse that college 2 faces (as it may accept a reject of college 1 ).

For a fixed admission standard, we want to ensure that very high quality students are almost never rejected, and very poor students are almost always rejected. For this, we assume that for a fixed signal $\sigma$, we have $G(\sigma \mid x) \rightarrow 0$ as $x \rightarrow \infty$ and $G(\sigma \mid x) \rightarrow 1$ as $x \rightarrow 0$. For instance, exponentially distributed signals have this property $G(\sigma \mid x)=$ $1-e^{-\sigma / x}$. More generally, this obtains for signals drawn from any "location family", in which the conditional cdf of signals $\sigma$ is given by $G((\sigma-x) / \mu)$, for any smooth cdf $G$ and $\mu>0-$ e.g. normal, logistic, Cauchy, or uniformly distributed signals. The strict MLRP then holds if the density is strictly $\log$-concave, i.e., $\log G^{\prime}$ is strictly concave.

C. Equilibrium. We consider a simultaneous move game by colleges and students. This yields the same equilibrium prediction as when students move first, as they are atomless. 10 An equilibrium is a triple $\left(S^{*}(\cdot), \underline{\sigma}_{1}^{*}, \underline{\sigma}_{2}^{*}\right)$ such that:

(a) Given $\left(\underline{\sigma}_{1}^{*}, \underline{\sigma}_{2}^{*}\right), S^{*}(x)$ is an optimal college application portfolio for each $x$,

(b) Given $\left(S^{*}(\cdot), \underline{\sigma}_{j}^{*}\right)$, college $i$ 's payoff is maximized by admissions standard $\underline{\sigma}_{i}^{*}$.

We also wish to preclude trivial equilibria in our model in which one or both colleges reject everybody with a very high admissions threshold and students do not apply there.

\footnotetext{
${ }^{10}$ See Appendix A.2 Alternatively, colleges could move first, committing to an admission standard. This is arguably not the case, but regardless, it too yields the same equilibrium properties until we study affirmative action (proof omitted). In the interests of a unified treatment throughout the paper, we proceed in the simultaneous move world.
} 
A robust equilibrium also requires that any college that expects to have excess capacity set the lowest admissions threshold. Since $\kappa_{1}+\kappa_{2}<1$, both colleges will have applicants.

In a robust sorting equilibrium, colleges' and students' strategies are monotone. This means that the better college is more selective $\left(\underline{\sigma}_{1}^{*}>\underline{\sigma}_{2}^{*}\right)$ and higher caliber students are increasingly aggressive in their portfolio choice: The weakest apply nowhere; better students apply to the "easier" college 2; even better ones "gamble" by applying also to college 1 ; the next tier up applies to college 1 while shooting an "insurance" application to college 2 ; finally, the top students confidently just apply to college 1 . Monotone strategies ensure the intuitive result that the distribution of student calibers at college 1 first-order stochastically dominates that of college 2 (see Claim 3 in Appendix A.7), so that all top student quantiles are larger at college 1 . This is the most compelling notion of student sorting in our environment with noise (Chade, 2006).

Our concern with a robust sorting equilibrium may be motivated on efficiency grounds. If there are complementarities between student caliber and college quality, so that welfare is maximized by assigning the best students to the best colleges, any decentralized matching system must necessarily satisfy sorting to be (constrained) efficient. Since formalizing this idea would add notation and offer little additional insight, we have abstracted from these normative issues and focused on the positive analysis of the model.

D. Common versus Private Values. Notice that in our model colleges care about the true caliber of a student and not about the signal per se. In other words, the model exhibits common values on the side of the colleges. Appendix A.1 shows colleges behave in exactly the same way if they do not care about caliber but care only about the signal i.e. the private values case. In this interpretation, students differ in their observables $x$ (known to both students and colleges), and also in their "fit" for each college $\epsilon_{i}$ (known only to the college). College payoffs depend on both observables and fit through the signal $\sigma_{i} \equiv \sigma_{i}\left(x, \epsilon_{i}\right)$. Until the affirmative action application in Section 7 , all the results apply to the private values case as well, albeit with a different interpretation.

\section{Equilibrium Analysis for Students}

\subsection{The Student Optimization Problem}

We begin by solving for the optimal college application set for a given pair of admission chances at the two colleges. Consider the portfolio choice problem for a student with 
admission chances $0 \leq \alpha_{1}, \alpha_{2} \leq 1$. The expected payoff of applying to both colleges is $\alpha_{1} v+\left(1-\alpha_{1}\right) \alpha_{2} u$. The marginal benefit $M B_{i j}$ of adding college $i$ to a portfolio of college $j$ is then:

$$
\begin{aligned}
M B_{21} & \equiv\left[\alpha_{1} v+\left(1-\alpha_{1}\right) \alpha_{2} u\right]-\alpha_{1} v=\left(1-\alpha_{1}\right) \alpha_{2} u \\
M B_{12} & \equiv\left[\alpha_{1} v+\left(1-\alpha_{1}\right) \alpha_{2} u\right]-\alpha_{2} u=\alpha_{1}\left(v-\alpha_{2} u\right)
\end{aligned}
$$

The optimal application strategy is then given by the following rule:

(a) Apply nowhere if costs are prohibitive: $c>\alpha_{1} v$ and $c>\alpha_{2} u$.

(b) Apply just to college 1 , if it beats applying just to college $2\left(\alpha_{1} v \geq \alpha_{2} u\right)$, and nowhere $\left(\alpha_{1} v \geq c\right)$, and to both colleges $\left(M B_{21}<c\right.$, i.e. adding college 2 is worse).

(c) Apply just to college 2, if it beats applying just to college $1\left(\alpha_{2} u \geq \alpha_{1} v\right)$, and nowhere $\left(\alpha_{2} u \geq c\right)$, and to both colleges $\left(M B_{12}<c\right.$, i.e. adding college 1 is worse).

(d) Apply to both colleges if this beats applying just to college $1\left(M B_{21} \geq c\right)$, and just to college $2\left(M B_{12} \geq c\right)$, for then, these solo application options respectively beat applying to nowhere, as $\alpha_{1} v>M B_{12} \geq c$ and $\alpha_{2} u>M B_{21} \geq c$ by (1)-(2).

This optimization problem admits an illuminating and rigorous graphical analysis. The left panel of Figure 2 depicts three critical curves: $M B_{21}=c, M B_{12}=c, \alpha_{1} v=\alpha_{2} u$. All three curves share a crossing point, since $M B_{21}=M B_{12}$, when $\alpha_{1} v=\alpha_{2} u$.

Cases $(a)-(d)$ partition the unit square into $\left(\alpha_{1}, \alpha_{2}\right)$ regions corresponding to the portfolio choices $(a)-(d)$, denoted $\Phi, C_{2}, B, C_{1}$, shaded in the right panel of Figure 2 , This summarizes the portfolio choice of a student with any admissions chances $\left(\alpha_{1}, \alpha_{2}\right)$.

In the marginal improvement algorithm of Chade and Smith (2006), a student first decides whether she should apply anywhere. If so, she asks which college is the best singleton. In Figure 2 at the left, college 1 is best right of the line $\alpha_{1} v=\alpha_{2} u$, and college 2 is best left of it. Next, she asks whether she should apply anywhere else. Intuitively, there are two distinct reasons for applying to both colleges that we can now parse: Either college 1 is a stretch school — i.e., a gamble, added as a lower-chance higher payoff option - or college 2 is a safety school, added for insurance. In Figure 2 , these are the parts of region $B$ above and below the line $\alpha_{1} v=\alpha_{2} u$, respectively.

The choice regions obey some natural comparative statics. The application region $C_{i}$ to either college increases in its payoff, in light of expressions (1) and (2), and the 

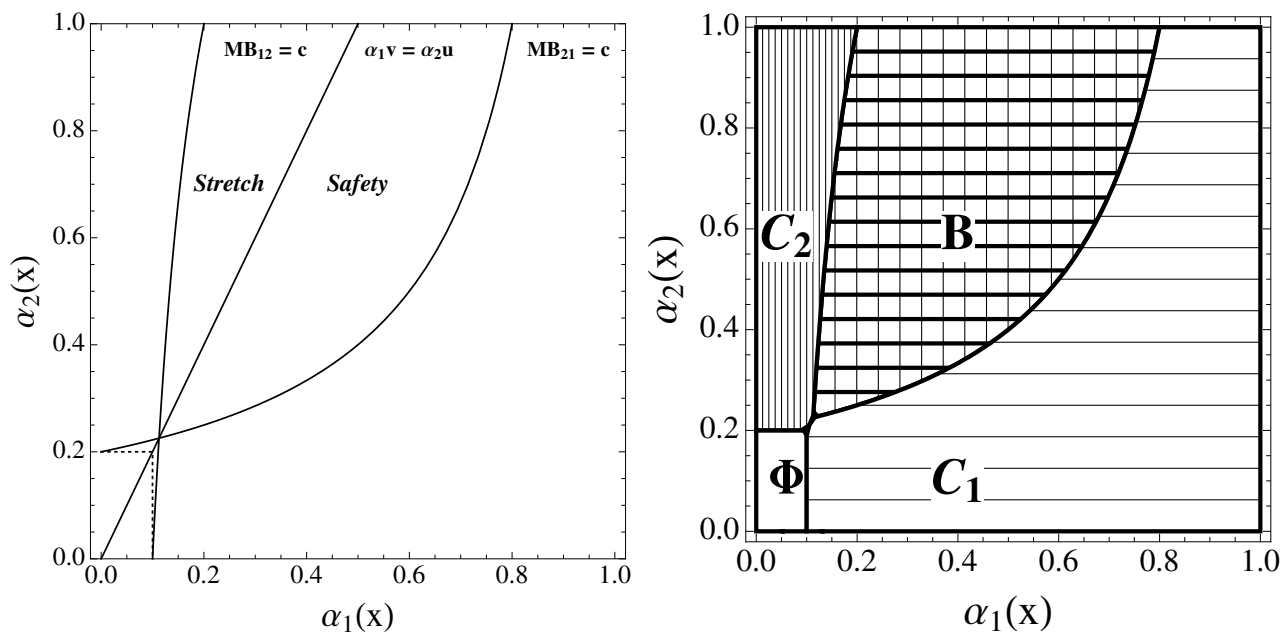

Figure 2: Optimal Decision Regions. The left panel depicts $(i)$ a dashed box, inside which applying anywhere is dominated; ( $i i)$ the indifference line for solo applications to colleges 1 and 2; and (iii) the marginal benefit curves $M B_{12}=c$ and $M B_{21}=c$ for adding colleges 1 or 2 . The right panel shows the optimal application regions. A student in the blank region $\Phi$ does not apply to college. He applies to college 2 only in the vertical shaded region $C_{2}$; to both colleges in the hashed region $B$, and to college 1 only in the horizontal shaded region $C_{1}$.

region $B$ expands rightwards in the college 2 payoff $u$, and leftwards in the college 1 payoff $v$. In particular, if a student enjoys fixed acceptance rates at the two colleges, a college grows less attractive as the payoff of its rival rises. Also, as the application cost $c$ rises, the joint application region $B$ shrinks and the empty set $\Phi$ grows.

Although outside our model, let us briefly consider non-linear costs - for instance, the second application costs may be less than $c$, possibly due to some duplication of forms, essays, etc. We analyze this in the online appendix, and show that region $B$ is bigger and the remaining regions smaller than with constant costs. Interestingly, some types who would send no singleton applications would nonetheless apply to both colleges.

\subsection{Admission Chances and Student Calibers}

We have solved the optimization for known acceptance chances. But we wish to predict the portfolio decisions of the students, despite the endogenous acceptance chances. To this end, we now derive a mapping from student types to student application portfolios. Fix the thresholds $\underline{\sigma}_{1}$ and $\underline{\sigma}_{2}$ set by college 1 and college 2. Student $x$ 's acceptance chance at college $i, i=1,2$, is given by $\alpha_{i}(x) \equiv 1-G\left(\underline{\sigma}_{i} \mid x\right)$. Since a higher caliber student generates stochastically higher signals, $\alpha_{i}(x)$ increases in $x$. In fact, it is a smoothly monotone onto function - namely, it is strictly increasing and differentiable, 


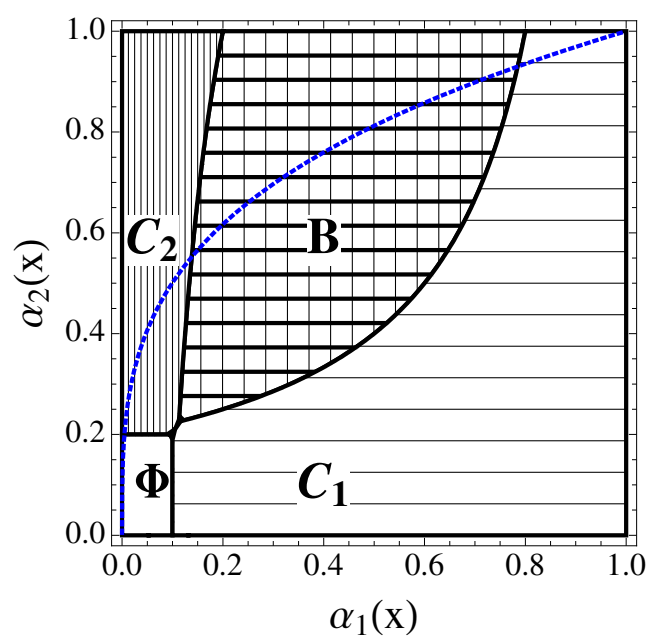

Figure 3: The Acceptance Function with Exponential Signals. The figure depicts the acceptance function $\psi\left(\alpha_{1}\right)$ for the case of exponential signals. As their caliber increases, students apply to nowhere $(\Phi)$, college 2 only $\left(C_{2}\right)$, both colleges $(B)$ - specifically, first using college 1 as a stretch school, and later college 2 as a safety school - and finally college 1 only $\left(C_{1}\right)$. Student behavior is therefore monotone for the acceptance function depicted.

with $0<\alpha_{i}(x)<1$, and the limit behavior $\lim _{x \rightarrow 0} \alpha_{i}(x)=0$ and $\lim _{x \rightarrow \infty} \alpha_{i}(x)=1$.

Taking the acceptance chances as given, each student of caliber $x$ faces the portfolio optimization problem of $\$ 3.1$. She must choose a set $S(x)$ of colleges to apply to, and accept the offer of the best school that admits her. We now translate the sets $\Phi, C_{2}, B, C_{1}$ of acceptance chance vectors into corresponding sets of calibers, namely, $\Phi, \mathcal{C}_{2}, \mathcal{B}, \mathcal{C}_{1}$.

Key to our graphical analysis is a quantile-quantile function relating student admission chances at the colleges: Since $\alpha_{i}(x)$ strictly rises in the student's type $x$, a student's admission chance $\alpha_{2}$ to college 2 is strictly increasing in his admission chance $\alpha_{1}$ to college 1. Inverting the admission chance in the type $x$, the inverse function $\xi(\alpha, \sigma)$ is the student type accepted with chance $\alpha$ given the admission standard $\sigma$, namely $\alpha \equiv 1-G(\sigma \mid \xi(\alpha, \sigma))$. This yields an implied differentiable acceptance function

$$
\alpha_{2}=\psi\left(\alpha_{1}, \underline{\sigma}_{1}, \underline{\sigma}_{2}\right)=1-G\left(\underline{\sigma}_{2} \mid \xi\left(\alpha_{1}, \underline{\sigma}_{1}\right)\right)
$$

We prove in the appendix that the acceptance function rises in college 1's standard $\underline{\sigma}_{1}$ and falls in college 2's standard $\underline{\sigma}_{2}$, and tends to 0 and 1 as thresholds near extremes.

By Figure 3, secant lines drawn from the origin or $(1,1)$ to successive points along the acceptance function decrease in slope. To this end, say that a function $h:[0,1] \rightarrow[0,1]$ has the double secant property if $h(\alpha)$ is weakly increasing on $[0,1]$ with $h(0)=0$, 
$h(1)=1$, and the two secant slopes $h(\alpha) / \alpha$ and $(1-h(\alpha)) /(1-\alpha)$ are monotone in $\alpha$. This description fully captures how our acceptance chances relate to one another.

Theorem 1 (The Acceptance Function) The acceptance function $\alpha_{2}=\psi\left(\alpha_{1}\right)$ has the double secant property. Conversely, for any smooth monotone onto function $\alpha_{1}(x)$, and any $h$ with the double secant property, there exists a continuous signal density $g(\sigma \mid x)$ with the strict MLRP, and thresholds $\underline{\sigma}_{1}, \underline{\sigma}_{2}$, for which admission chances of student $x$ to colleges 1 and 2 are $\alpha_{1}(x)$ and $h\left(\alpha_{1}(x)\right)$.

This result gives a complete characterization of how student admission chances at two ranked universities should compare. It says that if a student is so good that he is guaranteed to get into college 1, then he is also a sure bet at college 2; likewise, if he is so bad that college 2 surely rejects him, then college 1 follows suit. More subtly, we arrive at the following testable implication about college acceptance chances:

Corollary 1 As a student's caliber rises, the ratio of his acceptance chances at college 1 to college 2 rises, as does the ratio of his rejection chances at college 2 to college 1.

For an example, suppose that caliber signals have the exponential density $g(\sigma \mid x)=$ $(1 / x) e^{-\sigma / x}$. The acceptance function is then given by the increasing and concave geometric function $\psi\left(\alpha_{1}\right)=\alpha_{1}^{\underline{\sigma}_{2} / \underline{\sigma}_{1}}$, as seen in Figure 3 (as long as college 2 has a lower admission standard). The acceptance function is closer to the diagonal when signals are noisier, and farther from it with more accurate signals ${ }^{11}$ For an extreme case, as we approach the noiseless case, a student is either acceptable to neither college, both colleges, or just college 2 (assuming that it has a lower admission standard). The $\psi$ function tends to a function passing through $(0,0),(0,1)$, and $(1,1){ }^{12}$

Since a student's decision problem is unchanged by affine transformations of costs and benefits, we henceforth assume a payoff $v=1$ of college 1 ; so, college 2 pays $u \in(0,1)$. Throughout the paper, we also realistically assume that application costs are not too high relative to the college payoffs - specifically, $c<u(v-u) / v=u(1-u)$ and $c<u / 4$. The first inequality guarantees that the curves $M B_{21}=c$ and $M B_{12}=c$ do not cross twice inside the unit square 13 The second inequality ensures that the $M B_{21}=c$ curve crosses

\footnotetext{
${ }^{11}$ Specifically, for the earlier location-scale family, $\psi\left(\alpha_{1}\right)$ rises in the signal accuracy $1 / \mu$ (see Persico (2000)). Easily, the acceptance function tends to the top of the box in Figure 3 as $\mu \rightarrow 0$, since $\psi\left(\alpha_{1}\right)=1-G(-\infty)=1$, and to the diagonal $\psi\left(\alpha_{1}\right)=1-G\left(0+G^{-1}\left(1-\alpha_{1}\right)\right)=\alpha_{1}$ as $\mu \rightarrow \infty$.

${ }^{12}$ The limit function is not well-defined: If a student's type is known, just these three points remain.

${ }^{13}$ For if $\alpha_{2}=1$, then $M B_{21}=c$ and $M B_{12}=c$ respectively force $\alpha_{1}=1-(c / u)$ and $\alpha_{1}=c /(v-u)$. Now, $1-(c / u)>c /(v-u)$ exactly when $c<u(1-u) / v$.
} 
below the diagonal.14 If either inequality fails, the analysis trivializes since multiple college applications are impossible for some acceptance functions, as they are too costly.

\section{Equilibrium Analysis for Colleges}

\subsection{A Supply and Demand Approach}

Each college $i$ chooses an admission standard $\underline{\sigma}_{i}$ as a best response to its rival's threshold $\underline{\sigma}_{j}$ and the student portfolios. With a continuum of students, the resulting enrollment $\mathcal{E}_{i}$ at colleges $i=1,2$ is a non-stochastic number (recall that $\alpha_{i}(x) \equiv 1-G\left(\underline{\sigma}_{i} \mid x\right)$ ):

$$
\begin{aligned}
& \mathcal{E}_{1}\left(\underline{\sigma}_{1}, \underline{\sigma}_{2}\right)=\int_{\mathcal{B}_{\mathcal{C}}} \alpha_{1}(x) f(x) d x \\
& \mathcal{E}_{2}\left(\underline{\sigma}_{1}, \underline{\sigma}_{2}\right)=\int_{\mathcal{C}_{2}} \alpha_{2}(x) f(x) d x+\int_{\mathcal{B}} \alpha_{2}(x)\left(1-\alpha_{1}(x)\right) f(x) d x,
\end{aligned}
$$

suppressing the dependence of the sets $\mathcal{B}, \mathcal{C}_{1}$ and $\mathcal{C}_{2}$ on the student application strategy. To understand (4) and (5), observe that caliber $x$ student is admitted to college 1 with chance $\alpha_{1}(x)$, to college 2 with chance $\alpha_{2}(x)$, and finally to college 2 but not college 1 with chance $\alpha_{2}(x)\left(1-\alpha_{1}(x)\right)$. Also, anyone that college 1 admits will enroll automatically, while college 2 only enrolls those who either did not apply or got rejected from college 1 .

If we substitute optimal student portfolios into the enrollment equations (4)-(5), then they behave like demand curves where the admissions standards are the prices. Our framework affords analogues to the substitution and income effects in demand theory. The admission rate of any college obviously falls in its anticipated admission standard the standards effect. But there is a compounding portfolio effect - that enrollment also falls due to an application portfolio shift. Each college's applicant pool shrinks in its own admissions threshold. We then deduce in the appendix the "law of demand": If a college raises its admission standard, then its enrollment falls. Because of our portfolio effect, a college faces a more elastic demand for slots than predicted purely by the standards effect. A lower admission bar will invite applications from new students 15

The law of demand applies outside the two college setting. For intuition, suppose that the admissions standard at a college rises. Absent any student portfolio changes, fewer

\footnotetext{
${ }^{14}$ For $M B_{21}=c$ has no roots on the diagonal $\alpha_{2}=\alpha_{1}$ if $c>u / 4$.

${ }^{15}$ The portfolio effect may act with a lag — for instance, a college may unexpectedly ease admission standards one year, and see their applicant pool expand the next year when this becomes understood.
} 
students meet its tougher admission threshold (the standards effect), and its enrollment falls. The portfolio adjustment reinforces this effect. Those who had marginally chosen to add this college to their portfolios now excise it (the portfolio effect).

In consumer demand theory, the "price" of one good affects the demand for the other, and in the two good world, they are substitutes. Analogously, we prove in the appendix, that a college's enrollment demand rises in its rival's admission standard. This owes to a portfolio spillover effect. If it grows tougher to gain admission to college $i$, then those who only applied to its rival continue to do so, some who were applying to both now apply just to $j$ (which helps college $j$ when it is the lesser school), and also some at the margin who applied just to $i$ now also add college $j$ to their portfolios ${ }^{16}$

Since capacities imply vertical supply curves, we have justified a supply and demand analysis, in which the colleges are selling differentiated products. Ignoring for now the possibility that some college might not fill its capacity, equilibrium without excess capacity requires that both markets clear:

$$
\kappa_{1}=\mathcal{E}_{1}\left(\underline{\sigma}_{1}, \underline{\sigma}_{2}\right) \quad \text { and } \quad \kappa_{2}=\mathcal{E}_{2}\left(\underline{\sigma}_{1}, \underline{\sigma}_{2}\right)
$$

Since each enrollment (demand) function is falling in its own threshold, we may invert these equations. This yields for each school $i$ the threshold that "best responds" to its rival's admissions threshold $\underline{\sigma}_{j}$ so as to fill their capacity $\kappa_{i}$ :

$$
\underline{\sigma}_{1}=\Sigma_{1}\left(\underline{\sigma}_{2}, \kappa_{1}\right) \quad \text { and } \quad \underline{\sigma}_{2}=\Sigma_{2}\left(\underline{\sigma}_{1}, \kappa_{2}\right)
$$

Given the discussion of the enrollment functions, we can treat $\Sigma_{i}$ as a "best response function" of college $i$. It rises in its rival's admission standard and falls in its own capacity. That is, the admissions standards at the two colleges are strategic complements. Figure 4 depicts a robust equilibrium as a crossing of these increasing functions.

By way of contrast, observe that without noise or without application costs, the better college is completely insulated from the actions of its lesser rival $-\Sigma_{1}$ is vertical. The equilibrium analysis is straightforward, and there is a unique robust equilibrium. In either case, the applicant pool of college 1 is independent of what college 2 does. For when the application signal is noiseless, just the top students apply to college 1 . And

\footnotetext{
${ }^{16} \mathrm{As}$ in consumer theory, complementarity may emerge with three or more goods available. With ranked colleges 1,2 , and 3 , college 3 may be harmed by tougher admissions at college 1 , if this encourages enough applications at college 2 .
} 


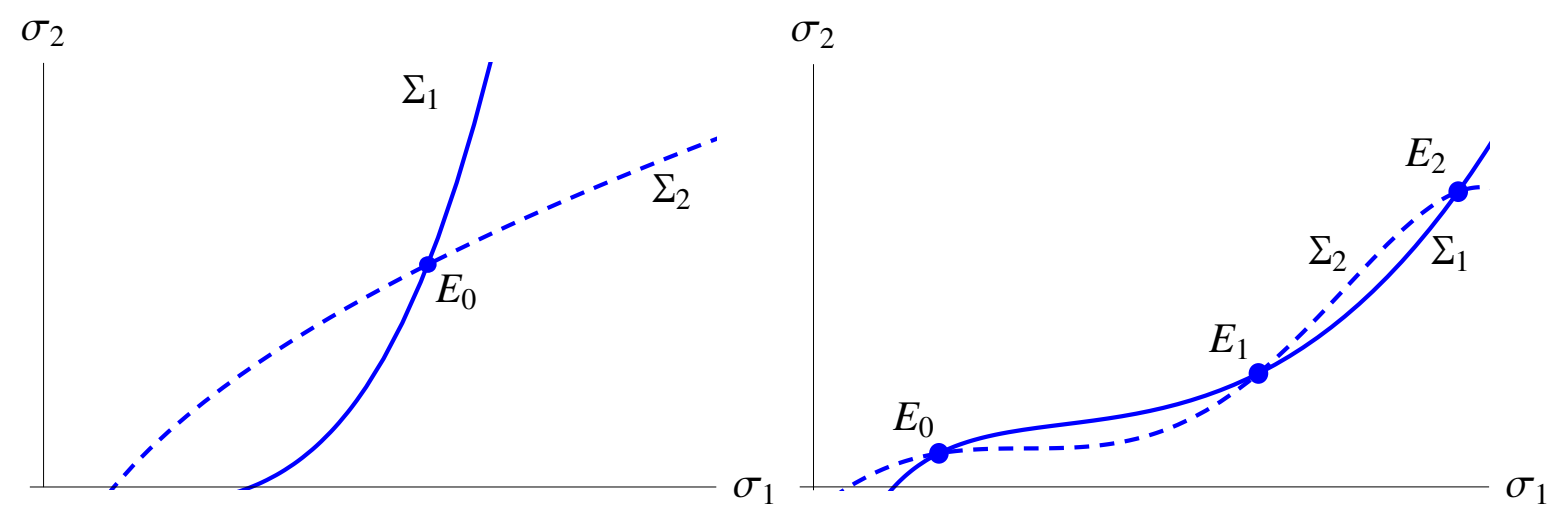

Figure 4: College Responses and Equilibria. In both panels, the functions $\Sigma_{1}$ (solid) and $\Sigma_{2}$ (dashed) give pairs of thresholds so that colleges 1 and 2 fill their capacities in equilibrium. The left panel depicts a unique robust stable equilibrium, while the right panel shows a case with multiple robust equilibria. $E_{0}$ and $E_{2}$ are stable, while $E_{1}$ is unstable.

when applications are free, all students apply to college 1, and will enroll if accepted.

With application costs and noise, $\Sigma_{1}$ is upward-sloping, as application pools depend on both college thresholds. When college 2 adjusts its admission standard, the student incentives to gamble on college 1 are affected. This feedback is critical in our paper. It leads to a richer interaction among the colleges, and perhaps to multiple robust equilibria.

In Figure 4, left panel, $\Sigma_{1}$ is steeper than $\Sigma_{2}$ at the crossing point. Let us call any such robust equilibrium stable. It is stable in the following sense: Suppose that whenever enrollment falls below capacity, the college eases its admission standards, and vice versa. Then this dynamic adjustment process pushes us back towards the equilibrium. Then at this theoretical level, admission thresholds act as prices in a Walrasian tatonnement. Unstable robust equilibria should be rare: They require that a college's enrollment responds more to the other school's admission standard than its own.

Theorem 2 (Existence) A robust stable equilibrium exists. College 1 fills its capacity. Also, there exists $\bar{\kappa}_{1}\left(\kappa_{2}, c\right)<1-\kappa_{2}$ satisfying $\lim _{c \rightarrow 0} \bar{\kappa}_{1}\left(\kappa_{2}, c\right)=1-\kappa_{2}$ such that if $\kappa_{1} \leq$ $\bar{\kappa}_{1}\left(\kappa_{2}, c\right)$, then college 2 also fills its capacity in any robust equilibrium. If $\kappa_{1}>\bar{\kappa}_{1}\left(\kappa_{2}, c\right)$, then college 2 has excess capacity in some robust equilibrium.

Surprisingly, college 2 may have excess capacity in equilibrium, despite excess demand for college slots ${ }^{17}$ This possibility is a consequence of portfolio effects: if college 1 is sufficiently big its standards may be low enough that college 2 fails to attract enough

\footnotetext{
17 College 1 cannot have excess capacity in a robust equilibrium. For then it must set the lowest possible standards, whereupon all students would apply and be accepted, violating its capacity constraint.
} 
applicants to fill its capacity even if it accepts all of them. In this case, $\alpha_{2}=1$ for all students, and so the acceptance function traverses the top side of the unit square in Figure 3. So as student caliber rises, the lowest students apply to college $\{2\}$, higher students to both colleges, and the best students just apply to college $\{1\}$. Let us observe in passing that this is a robust sorting equilibrium.

Since admissions standards are strategic complements, multiple robust equilibria are possible (right panel of Figure 4). $1^{18}$ In such a scenario, both colleges raise their standards, yet students send even more applications, and another robust equilibrium arises.

\subsection{Comparative Statics}

We now continue to explore the supply and demand metaphor, and derive some basic comparative statics. The potential multiplicity of robust equilibria makes a comparative statics exercise difficult. But fortunately, our analysis applies to all robust stable sorting equilibria and in some cases to all robust stable equilibria. Indeed, at any robust stable equilibrium, greater capacity at either college lowers both college admissions thresholds. This result speaks to the equilibrium effects at play. Greater capacity at one school, or an exogenous increase in the "supply" of slots at that college, reduces the "price" (admission standard) at both schools. The left panel of Figure 5 proves this assertion for an increase in $\kappa_{2}$, and the proof for a change in $\kappa_{1}$ is analogous.

For intuition, consider a robust stable sorting equilibrium, where students apply as in Figure 3. Let college 2 raise its capacity $\kappa_{2}$. For any admission standard $\underline{\sigma}_{1}$, this depresses $\underline{\sigma}_{2}$, so $\Sigma_{2}$ shifts down. Then the marginal student that was indifferent between applying to college 2 only $\left(C_{2}\right)$ and both colleges $(B)$ now prefers to apply to college 2 only. So fewer apply to college 1. Given this portfolio shift, college 1 drops its admission standards, and both thresholds are lower in the new equilibrium $E_{1}$. The same logic generates the analogous comparative static for an increase in capacity at college 1.

Unlike college capacity, changes in college payoffs or application costs affect both best response functions $\Sigma_{1}$ and $\Sigma_{2}$. As a result, the comparative statics can be ambiguous, and counter-intuitive results may emerge. For example, suppose the payoff $v$ of college 1 rises (right panel of Figure 5). At the current admissions standards, demand for college 1 will increase, while demand for college 2 decreases as more of its applicants gamble up on college 1 . These forces lead to new best response functions, namely a rightward shift

\footnotetext{
${ }^{18}$ The online appendix contains a solved example with multiplicity.
} 

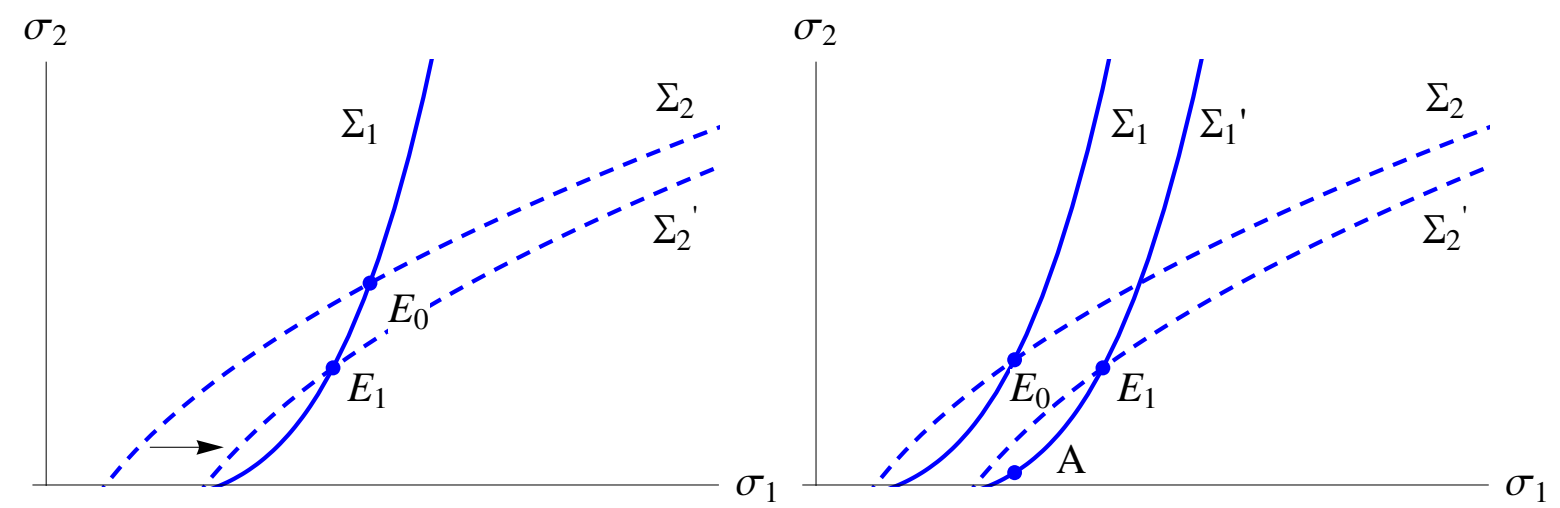

Figure 5: Comparative Statics. In both panels, the best response functions $\Sigma_{1}$ (solid) and $\Sigma_{2}$ (dashed) are drawn. The left panel considers a rise in $\kappa_{2}$, which shifts $\Sigma_{2}$ up and has no effect on $\Sigma_{1}$. In the right panel, we illustrate the effect of an increase in college 1's payoff, which shifts $\Sigma_{1}$ to the right and shifts $\Sigma_{2}$ down.

in $\Sigma_{1}$ and a downward shift in $\Sigma_{2}$.

At first glance, this has ambiguous effects: depending on the size of the shifts, both admissions standards could rise or both could fall, or the standard at college 1 could rise and that of college 2 could fall 19 But provided $\Sigma_{2}$ does not fall below the point $A$ in Figure 5, the new curves $\Sigma_{1}^{\prime}$ and $\Sigma_{2}^{\prime}$ will cross above and to the right of $A$. In that case, there is another robust and stable equilibrium in which $\underline{\sigma}_{1}$ rises. Notice that college 2 attracts more applications and admits more students at $A$ than at $E_{0}$ because of its lower standards, while losing joint admits at the same rate as before (see the proof of Theorem 3). So it must have excess demand at $A$, and thus $\Sigma_{2}^{\prime}$ must pass between $E_{0}$ and $A$, implying a new equilibrium $E_{1}$ in which college 1's standards increase.

Next, assume that the application cost $c$ rises, perhaps due to a rise in the SAT or ACT cost, or the common application fee. This has two effects. On the one hand, it decreases the number of college applications (the region $B$ in Figure 3 shrinks). This has an unambiguously negative effect on demand at both colleges. On the other hand, it decreases competition between the colleges, as there will be fewer overlapping applications. This has no effect on college 1 (since they always beat college 2 for joint admits), but it improves the yield of college 2 . As a result, demand at college 1 falls and $\Sigma_{1}$ shifts left, but the effect on $\Sigma_{2}$ is ambiguous.

Using an argument similar to the one above, we can show that there exists a new robust stable equilibrium in which $\underline{\sigma}_{1}$ falls. But due to the competition effect, we cannot

\footnotetext{
${ }^{19} \mathrm{We}$ face the same ambiguity in analyzing the effect of an increase in the payoff $u$ of college 2.
} 
be sure how $\underline{\sigma}_{2}$ moves: college 2 may raise its standards in the new equilibrium, if the higher applications costs deter sufficiently many students from gambling up.

Consider instead a rise in just one college's application cost, such as a college requiring a longer essay or imposing a greater fee. We argue that in a robust stable sorting equilibrium, if the application cost at either college slightly falls, then the admission standard at college 1 rises and its student caliber distribution stochastically worsens.

For example, if the application cost at college 2 falls, then more students apply, and it is forced to raise its standards. The marginal benefit of a stretch application to college 1 thus rises. To counter this, college 1 responds with a higher standard. Still, its set of applicants is of lower caliber than before (in the sense of the strong set order), and though it screens them more tightly, its caliber distribution stochastically worsens. By contrast, college 2 loses not only its worst students, but also top ones for whom it was insurance, and its caliber change is ambiguous.

Summarizing our results on changes in college payoffs and application costs:

Theorem 3 (Comparative Statics) In a robust stable sorting equilibrium:

(a) When $v$ increases, there exists another robust equilibrium in which $\underline{\sigma}_{1}$ increases.

(b) When c increases, there exists another robust equilibrium in which $\underline{\sigma}_{1}$ decreases.

(c) When either college's application cost increases marginally, $\underline{\sigma}_{1}$ decreases.

Whenever $\underline{\sigma}_{1}$ decreases due to one of the above changes, the distribution of enrolled calibers at college 1 improves in the sense of first order stochastic dominance.

The final part of the theorem suggests that top-tier colleges have an incentive to increase application costs, since this leads the weakest applicants to self-select out of applying to them. There is some evidence of this: many top-tier colleges require idiosyncratic essays as part of their application, effectively raising application costs 20 Yet this result relies on our assumption that students know their type and colleges do not, for if colleges were better than students at identifying caliber, then they might want to encourage applications by lowering application costs. This appears to be true for lowincome students: Hoxby and Avery (2012) show that many low-income high-achievers act as if they were unaware of their caliber, and do not apply to any selective colleges. In this case top schools should decrease frictions for low-income students, through application cost waivers and targeted recruiting efforts - both of which we see in practice.

\footnotetext{
${ }^{20}$ For example, one essay prompt from the University of Chicago this year is the Winston Churchill quote that "A joke is a very serious thing"; also, almost all of Amherst's essay prompts are based on quotes from Amherst professors and alumni.
} 
The logic underlying this section does not essentially depend on the assumption that there are two colleges. For instance, whenever colleges have overlapping applicant pools, a rise in capacity at any one college depresses the admission standards at all of them.

Consider the positive theory of this section in light of Hoxby (2009). She shows that during 1962-2007, the median college has become significantly less selective, while at the same time, admissions have become more competitive at the top $10 \%$ of colleges. Her explanation for the fall in standards hinges on capacity: the number of freshman places per high-school graduate has been rising steadily. But as we illustrate in Figure 5 , higher capacity at all schools should depress standards at all schools, via our spillover effect. As a countervailing force, she argues that students have simultaneously become more willing to enroll far from home, raising the relative payoff of selective colleges. This aligns with Theorem 3; starting at a robust and stable sorting equilibrium, a perceived increase in the value of an education at a top school leads it to raise its standards.

\section{Do Colleges and Students Sort in Equilibrium?}

Casual empiricism suggests that the best students apply to the best colleges, and those colleges are in turn the most selective. This logic justifies ranking colleges based on their admissions standards. Curiously, these claims are false without stronger assumptions. We identify and explore two possible types of sorting violations.

The first violation occurs when some relatively high calibers "play it safe". By Corollary 1, along the acceptance function, higher types enjoy a higher ratio of admissions chances at college 1 to college 2. But this does not imply a higher marginal benefit $\alpha_{1}\left(1-\alpha_{2} u\right)$ of applying to college 1 , and so lower types may apply more aggressively. We illustrate this in the left panel of Figure 6, where application sets are $\Phi,\{2\},\{1,2\},\{2\},\{1,2\},\{1\}$ as caliber rises. A concrete example is the Texas top $10 \%$ plan, which guarantees automatic admission to any school in the UT system for students graduating in the top $10 \%$ of their high-school class. Such students have little incentive to apply to slightly better out-of-state schools (college 1), since the payoff increment is small and they don't need the insurance of a second application. But students who just

miss the $10 \%$ cutoff may want the insurance, and so one might see more aggressive application portfolios from those (lower-caliber) students, generating a non-monotonicity.

The second violation occurs when the worse college sets a higher admissions standard. To see how this can happen, consider the edge case where the standards are the 

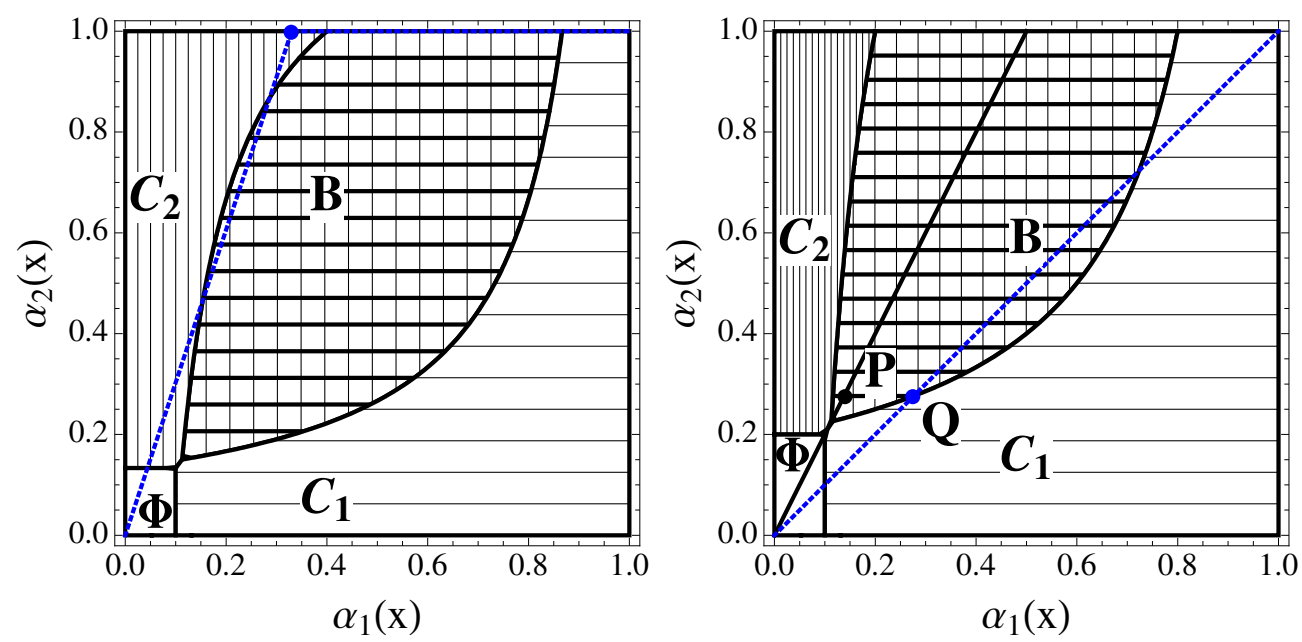

Figure 6: Non-Monotone Behavior. In the left panel, the signal structure induces a piecewise linear acceptance function. Student behavior is non-monotone, since there are both low and high caliber students who apply to college 2 only $\left(C_{2}\right)$, while intermediate ones insure by applying to both. In the right panel, equal thresholds at both colleges induce an acceptance function along the diagonal, $\alpha_{1}=\alpha_{2}$. Student behavior is non-monotone, as both low and high caliber students apply to college 1 only $\left(C_{1}\right)$, while middling caliber students apply to both. Such an acceptance function also arises when caliber signals are very noisy.

same at both colleges. With common admissions chances $\alpha$, the marginal benefit of a safety application $(1-\alpha) \alpha u$ is not increasing in $\alpha$ (and thus not in caliber, either). The right panel of Figure 6 depicts one such case — where the application sets are $\Phi,\{1\},\{1,2\},\{1\}$ as caliber rises.In this case college 2 only attracts insurance applications. This can be an equilibrium outcome if college 2 is small enough (and by making it still smaller, college 2 can end up setting a higher standard than college 1).

To rule out the first sorting violation, it suffices that college 2 offer a low payoff $(u<0.5)$, so that the payoff increment of admission to college 1 is large. We show in the Appendix that this ensures that the marginal benefit of additionally applying to college is increasing in caliber. The second sort of violation cannot occur when college 1 sets a sufficiently higher admission standard than college 2. This happens when college 1 is sufficiently smaller than college 2 . The threshold capacity will depend on the model primitives: rival capacity, applications cost, payoff differential and signal structure.

\section{Theorem 4 (Non-Sorting and Sorting in Equilibrium)}

(a) If college 2 is "too good" (i.e., $u>0.5$ ), then there exists a continuous MLRP density $g(\sigma \mid x)$ that yields a robust stable equilibrium with non-monotone student behavior.

(b) If college 2 is small enough relative to college 1 , then college 2 sets a higher admissions 
standard than college 1 in some robust equilibrium.

(c) If college 1 is small enough relative to college 2, and college 2 is not too good (namely, $u \leq 0.5)$, then there are only robust sorting equilibria and no college has excess capacity.

The challenge in proving this theorem is to show that all of non-monotone behavior outlined above can happen in equilibrium. For part $(a)$, we construct a robust nonmonotone equilibrium by starting with the acceptance function depicted in the left panel of Figure 6, which constrains the relationships between admissions chances across colleges to be some mapping $\alpha_{2}(x)=h\left(\alpha_{1}(x)\right)$. We then construct a particular acceptance chance $\alpha_{1}(x)$ so that the induced student behavior and acceptance rates given $\left(\alpha_{1}, h\left(\alpha_{1}\right)\right)$ equate college capacities and enrollments. Finally, we show that these two mappings satisfy the requirements of Theorem 1 and therefore can be generated by MLRP signals and monotone standards. For part (b), we show that that by perturbing a robust equilibrium with equal admissions chances by making college 2 smaller, we get a robust equilibrium with non-monotone standards. Finally, part (c) turns on showing that when $\kappa_{1}$ is relatively small, the crossing of the best-response functions must occur at a point where college 1 sets high enough standards that low caliber students don't apply there.

All told, parts $(a)$ and $(b)$ show that sorting may fail, which is surprising given how well behaved the signal structure is. Even in equilibrium, the optimal student portfolio may not increase with caliber; and worse colleges can enroll students of higher average caliber if they are sufficiently small 21 Since organizations like US News and World Report use statistics like the average SAT score of matriculants in their college rankings, this undercuts how colleges are ranked.

For an insightful counterpoint, consider what happens when students are limited to just a single application, as it is sometimes the case ${ }^{22}$ Recalling the left panel in Figure 2, we see that the diagonal line $\alpha_{1} u=\alpha_{2}$, and the individual rational equalities $\alpha_{2} u=\alpha_{1}=c$, jointly partition the application space into three relevant parts. But with any acceptance function with the falling secant property, low types apply nowhere, middle types apply to college 2, and high types apply to college 1. It should come as

\footnotetext{
${ }^{21}$ This can be illustrated using the right panel of Figure 6. Consider a robust equilibrium with equal admissions standards at the two colleges. If $f(x)$ concentrates most of its mass on the interval of low calibers who apply just to college 1 , then the average caliber of students enrolled at college 1 will be strictly smaller than that at college 2 . This example can be adjusted slightly so that college 2 is more selective than college 1. (See our online appendix for a fleshed out example of this phenomenon.)

${ }^{22}$ In Britain, all college applications go through UCAS, a centralized clearing house. A maximum of five applications is allowed.
} 
no surprise that there is a unique robust equilibrium. So it turns out that the sorting failures in Theorem $4(a)$ and $(b)$ require portfolio applications.

Moreover, the college non-monotonicity result requires that colleges have some "market power". To see this, suppose that there were instead two tiers of colleges, a top tier 1 and an lesser tier 2; each containing a continuum of otherwise identical colleges with total capacity $\kappa_{1}$ and $\kappa_{2}$ respectively. Students may apply to multiple colleges within a tier, each application generating a conditionally iid signal and costing $c$. Then college standards must be monotone; for if the top tier colleges were easier to get into, no student would ever apply to a second tier college. By contrast, we show in the online appendix that the student non-monotonicity result is robust to making the colleges atomistic.

\section{General Incomplete Information About Calibers}

A. When Students Do Not Know their Calibers. We have assumed that colleges observe conditionally independent evaluations of the students' true calibers. At the opposite extreme, one might envision a hypothetical world where colleges know student calibers, and students see noisy conditionally independent signals of them. Yet observe that this is informationally equivalent to a world in which students know their calibers, and colleges observe perfectly correlated signals. For any student sees a signal equal to $t+$ "noise", while both colleges see the student caliber $t$.

This embedding suggests that we could capture the world in which students and colleges alike only see noisy conditionally iid signals of calibers by relabeling the student signal as their caliber. We argue in the appendix that under this relabeling that world is a special case of the following one:

( $\star$ ) Students know their calibers and colleges observe affiliated noisy signals of them.

Thus, we can without loss of generality assume that students know their calibers and colleges vary by their signal affiliation 23 Observe that in this world, Theorem 1 remains a valid description of how the unconditional acceptance chances at the two colleges relate.

B. Perfectly Correlated Signals. This benchmark is highly instructive. Suppose first that the two colleges observe perfectly correlated signals of student calibers. As we mentioned above, this is akin to observing the caliber of each applicant. The key (counterfactual) feature here is that if a student is accepted by the more selective college,

\footnotetext{
${ }^{23}$ Some predictions outside of the model might differ in the known and unknown calibers cases.
} 
then so is he at the less selective one. This immediately implies that $\underline{\sigma}_{1}>\underline{\sigma}_{2}$ in equilibrium, for otherwise no one would apply to college 2 . So contrary to Theorem 4 , college behavior must be monotone in any equilibrium.

The analysis of this case differs in a few dimensions from 3.1 . Since $\underline{\sigma}_{1}>\underline{\sigma}_{2}$, applying to both colleges now yields payoff $\alpha_{1}+\left(\alpha_{2}-\alpha_{1}\right) u-2 c$. Unlike before. ${ }^{24}$

$$
M B_{21} \equiv\left(\alpha_{2}-\alpha_{1}\right) u=c \quad \text { and } \quad M B_{12} \equiv \alpha_{1}(1-u)=c
$$

because admission to college 1 guarantees admission to college 2 . In this informational world, both optimality equations are linear, and the latter is vertical (see Figure 7).

Assume that college 1 is sufficiently more selective than college 2 . Then the lowest caliber applicants apply to college 2 - namely, those whose admission chance exceeds $c / u$. Students so good that their admission chance at college 1 is at least $c /(1-u)$ add a stretch application, provided college 2 admits them with chance $c / u(1-u)$ or more. In Figure 7, this occurs when the acceptance function crosses above the intersection point of the curves $M B_{21}=c$ and $M B_{12}=c{ }^{25}$ Since the marginal benefit $M B_{12}$ is independent of the admission chance at college $2, M B_{12}>c$ for all higher calibers.

But monotone behavior for stronger caliber students requires another assumption. Consider the margin between applying just to college 1, or adding a safety application. The top caliber students will apply to college 1 only, since their admission chance is so high. But the behavior of slightly lesser student calibers is trickier, as the acceptance function can multiply cross the line $M B_{21}=c{ }^{26}$ Under slightly stronger assumptions, the acceptance function is concave; this precludes such perverse multiple-crossings, and implies monotone student behavior. ${ }^{27}$

Consider now the possibility of non-monotone student behavior. Absent a concave acceptance function, the previous sorting failure owing to multiple-crossings arises. But even with a concave acceptance function, a sorting failure arises if college 1 is not sufficiently choosier than college 2 . For then a suitably drawn concave acceptance function could consecutively hit regions $C_{1}, B$, then $C_{1}$, and a sorting failure ensues.

Having explored the impact of correlation on college-student sorting, we now flesh

\footnotetext{
${ }^{24}$ We suppress the caliber $x$ argument of the unconditional acceptance chance $\alpha_{i}(x)$ at college $i=1,2$.

${ }^{25}$ Namely, at the mutual intersection of regions $C_{1}, C_{2}$, and $B$ in Figure 7 . By inequality (13), this holds under our hypothesis that college 1 is sufficiently choosier than college 2 .

${ }^{26}$ For as seen in Figure 7 , that line also has a strictly falling secant.

${ }^{27}$ Concavity holds whenever $-G_{x}(\sigma \mid x)$ is log-supermodular. This is true when we further restrict to location families (like the Normal) or scale families (such as exponential).
} 

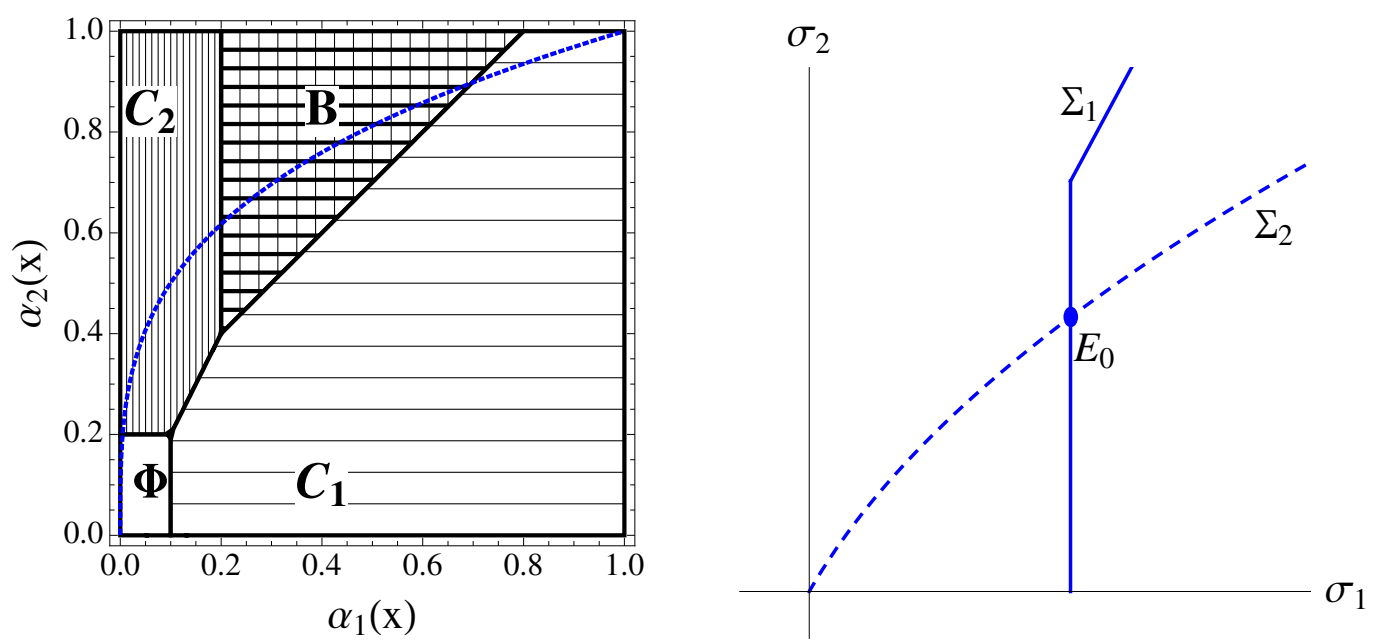

Figure 7: Student Behavior with Perfectly Correlated Signals. The shaded regions depict the optimal portfolio choices for students when colleges observe perfectly correlated signals. Unlike Figure 2, the $M B_{12}=c$ curve separating regions $B$ and $C_{2}$ is vertical. The $\Sigma_{1}$ curve at the right is vertical (up to a point), since college 2 no longer imposes an externality on college 1 when the set of calibers sending multiple applications is nonempty.

out its effect on college feedbacks. Since $M B_{12}$ is independent of the admission threshold at college 2 in (8), the pool of applicants to college 1 is unaffected by changes in $\underline{\sigma}_{2}$. Hence, the $\Sigma_{1}$ locus is vertical over most of its domain ${ }^{28}$ The better college is insulated from the decisions of its weaker rival, and the setting is not as rich as our baseline conditionally iid case. It is obvious from Figure 7 that the robust equilibrium is unique.

C. Affiliated College Evaluations. We now turn to the general case of assumption ( $\star$ ). Each student knows his caliber $x$, and colleges see signals $\sigma_{1}, \sigma_{2}$ of them, with an affiliated joint density $g\left(\sigma_{1}, \sigma_{2} \mid x\right) 29$ Since acceptance and rejection by college 1 is good and bad news, respectively, it intuitively follows that

$$
\alpha_{2}^{A} \geq \alpha_{2} \geq \alpha_{2}^{R}
$$

Here, $\alpha_{2}^{A}$ and $\alpha_{2}^{R}$ are the respective acceptance chances at college 2 given acceptance and rejection at college 1 . For instance, $1=\alpha_{2}^{A}>\alpha_{2}>\alpha_{2}^{R}$ with perfectly correlation. But in the conditionally iid case, college 2 is unaffected by the decision of college 1 , and

\footnotetext{
${ }^{28}$ The locus $\Sigma_{1}$ vertical if college 1 is sufficiently more selective than college 2 . For then the acceptance function is high enough that it traverses region $B$, and some students send multiple applications. If not, then the acceptance function could hit $C_{2}$ and then $C_{1}$, bipassing region $B$. In that case, the marginal applicant to college 1 depends on $\underline{\sigma}_{2}$, and hence $\Sigma_{1}$ is not vertical.

${ }^{29}$ As is standard, this means that $g$ obeys the monotone likelihood ratio property for every fixed $x$.
} 
so $\alpha_{2}^{A}=\alpha_{2}=\alpha_{2}^{R}$. Since these are intuitively opposite ends of an affiliation spectrum, we call evaluations more affiliated if the conditional acceptance chance $\alpha_{2}^{A}$ at college 2 is higher for any given unconditional acceptance chance $\alpha_{2}$.

Let's first see how affiliation affects student applications. In this more general setting,

$$
M B_{21}=\left(1-\alpha_{1}\right) \alpha_{2}^{R} u \quad \text { and } \quad M B_{12}=\alpha_{1}\left(1-\alpha_{2}^{A} u\right)
$$

This subsumes the marginal analysis for our conditionally iid and perfectly correlated cases: (1)-(2) and (8). Relative to these benchmarks, the acceptance curse (or the "acceptance blessing") conferred by college 1's two possible decisions lessens the marginal gain of an extra application to either college - due to inequality (9). More intuitively, double admission is more likely when signals are more affiliated. In our graph, this is reflected by a right shift of the curve $M B_{12}=c$, and a left shift of $M B_{21}=c$. In other words, for any given college admission standards, students send both fewer stretch and safety applications when college evaluations are more affiliated.

We next explore how affiliated evaluations affects college behavior. Consider the best reply locus $\Sigma_{1}$ of college 1 . It is upward-sloping with conditionally iid college evaluations, and vertical with perfectly correlated evaluations. We argue that it is upward-sloping with affiliated evaluations, and grows steeper as evaluations grow more affiliated. In other words, our benchmark conditionally iid case delivers robust results about two-way college feedbacks. Perfectly correlated evaluations therefore ignores the effect of the lesser on the better college, and so is less reflective of the affiliated case.

We first show that the best response curve $\Sigma_{1}$ slopes upward with imperfect affiliation. For let college 1's admission standard $\underline{\sigma}_{1}$ rise. Then its unconditional acceptance chance $\alpha_{1}$ falls for every student. The marginal student pondering a stretch application must then fall in order for college 1 to fill its capacity (6). Optimality $M B_{12}=c$ in (10) next requires that this student's conditional acceptance chance $\alpha_{2}^{A}$ fall. This only happens if his unconditional chance $\alpha_{2}$ falls too - i.e. the standard $\underline{\sigma}_{2}$ rises.

Next, college 1's best response curve $\Sigma_{1}$ slopes up more steeply when college evaluations are more strongly affiliated. For as affiliation rises, the marginal student sees a greater fall in his admission chance $\alpha_{2}^{A}$. So his unconditional chance $\alpha_{2}$ falls more too, and college 2's admission standard $\underline{\sigma}_{2}$ drops more than before (see Figure 7), as claimed. As an aside, since robust equilibrium is unique with perfectly correlated college evaluations, uniqueness intuitively holds more often when we are closer to this extreme.

Finally, we consider how the equilibrium sorting result Theorem 4 changes with affili- 
ation. By examining (10), we see that as we transition from conditionally iid to perfectly correlated signals with increasing affiliation, the region of multiple applications shrinks monotonically. This simple insight has important implications for sorting behavior. By standard continuity logic, for very low or high affiliation, sorting obtains and fails exactly as in the respective conditional independent or perfectly correlated cases. More strongly, the negative result in Theorem 4 (b) fails for moderately high affiliation: For then nonmonotone college behavior is impossible since the locus $M B_{21}=c$ in (8) lies strictly above the diagonal, and thus the same holds for 10 with sufficiently affiliated signals. So the acceptance function would lie below the diagonal if admission standards were inverted, and no student would ever apply to college 2. Lastly, the logic for the positive sorting result of Theorem $4(c)$ is still valid: both student and college behavior are monotone if college 2 is not too small and not too good, and if the acceptance function is concave - appealing to the logic for perfectly correlated signals.

\section{$7 \quad$ The Spillover Effects of Affirmative Action}

We now explore the effects of an affirmative action policy ${ }^{30}$ Slightly enriching our model, we first assume that a fraction $\phi$ of the applicant pool belongs to a target group. This may be an under-represented minority, but it may also be a majority group. For instance, many states favor their own students at state colleges - Wisconsin public colleges can have at most $25 \%$ out-of-state students. Just as well, some colleges strongly value athletes or students from low-income backgrounds. We assume a common caliber distribution, so that there is no other reason for differential treatment of the applicants.

Assume that students honestly report their "target group" status on their applications. Reflecting the colleges' desire for a more diverse student body, let college $i$ earn a bonus $\pi_{i} \geq 0$ for each enrolled target student. Colleges may set different thresholds for the two groups. If college $i$ offer a "discount" $\Delta_{i}$ to target applicants, then the respective standards for non-target and target groups are $\left(\underline{\sigma}_{1}, \underline{\sigma}_{2}\right)$ and $\left(\underline{\sigma}_{1}-\Delta_{1}, \underline{\sigma}_{2}-\Delta_{2}\right)$. Akin to third degree price discrimination, now colleges equate the shadow cost of capacity across groups for the marginally admitted student. So at each college, the expected payoff of the marginal admits from the two groups should coincide - except at a corner solution, when a college admits all students from a group. This yields two new equilibrium condi-

\footnotetext{
${ }^{30}$ For recent treatment of complementary affirmative action issues, see Epple, Romano, and Sieg (2008), Hickman (2010), Groen and White (2004), and Curs and Singell (2002).
} 
tions that account for the fact that ex post, colleges behave rationally, and equate their expected values of target and non-target applicants. Along with college market clearing (6), equilibrium entails solving four equations in four unknowns.

The analysis is simpler if we assume private values, and we begin with this case. Since colleges care directly about the signal observed with private values, equalization of the marginal admits of the two groups $i=1,2$ reduces to $\underline{\sigma}_{i}=\underline{\sigma}_{i}-\Delta_{i}+\pi_{i}$, and so $\Delta_{i}=\pi_{i}$. That is, the 'discount' afforded to a student from the target group equals the additional payoff a college enjoys from admitting a student from the target versus the non-target group. Thus, college preferences for target group students translate directly into admission standard discounts for that group.

Instead, with common values the equalization of shadow values (i.e., the expected payoff of the marginal admits from the two groups) yields the richer conditions:

$$
\begin{aligned}
E\left[X+\pi_{1} \mid \sigma=\underline{\sigma}_{1}-\Delta_{1}, \text { target }\right] & =E\left[X \mid \underline{\sigma}_{1}, \text { non-target }\right] \\
E\left[X+\pi_{2} \mid \sigma=\underline{\sigma}_{2}-\Delta_{2}, \text { target, accepts }\right] & =E\left[X \mid \underline{\sigma}_{2}, \text { non-target, accepts }\right]
\end{aligned}
$$

Here, $X$ is the student caliber. As before, along with (6), equilibrium amounts to solving four equations in four unknowns. Notice that no longer do we have $\Delta_{i}=\pi_{i}$, which significantly complicates the analysis of the problem. Rather, the discount $\Delta_{i}$ now depends on $\pi_{i}$ in a nonlinear fashion via the conditional expectations. To obtain a sharp result, we impose the following notion of stability, which we explain in the online appendix: when the shadow value of a target student exceeds that of a non-target student, college $i$ responds by raising the target advantage $\Delta_{i}$. Call the equilibrium shadow value stable if this dynamic adjustment process pushes us back to the equilibrium.

Theorem 5 (Affirmative Action) Fix $\pi_{1}=\pi_{2}=0$.

(a) Assume private values and a robust stable equilibrium. As the preference for a target group at one college rises, it favors those students and penalizes non-target students, with no effect on the other college. As the preference $\pi$ for target students at both colleges rises from $\pi_{1}=\pi_{2}=\pi=0$, both favor them and penalize non-target ones.

(b) Assume that $\Delta_{1}=\Delta_{2}=0$ is a robust shadow value stable equilibrium with monotone student behavior ${ }^{31}$ As the preference for a target group at college 1 rises, it favors those students and college 2 penalizes them. As the preference for target students at college 2 rises, both colleges favor them more.

\footnotetext{
${ }^{31}$ Such an equilibrium easily exists when $c=0$, and by continuity for $c$ small enough.
} 
Observe the indirect effect of student preferences: Nontarget students face stiffer admission standards since the shadow value of capacity is now higher. The assumption that $\pi_{1}=\pi_{2}=0$ is important, as it precludes some complex feedback effects that emerge when there is already a preference for target students at the outset.

Consider private values. For a sharper insight, let the signal distribution be $G(\sigma-x)$ (location family). Suppose that both colleges exhibit identical preference $\pi$ for the target group. Since $\Delta=\pi$ for both colleges, the acceptance relation is identical for both groups and given by $\psi\left(\alpha_{1}\right)=1-G\left(\underline{\sigma}_{2}-\underline{\sigma}_{1}+G^{-1}\left(1-\alpha_{1}\right)\right.$ ) (the discounts cancel out in the argument of $G$ for the target group). This implies that caliber $x$ from the target group applies exactly as if they were a type $x+\pi$ from the non-target group: a testable claim.

If instead only one college, say 1 , has a preference $\pi_{1}$ for the target group, then it sets a discount $\Delta_{1}=\pi_{1}$. The acceptance relation for the target group is then $\psi\left(\alpha_{2}\right)=$ $1-G\left(\underline{\sigma}_{2}-\underline{\sigma}_{1}+\Delta_{1}+G^{-1}\left(1-\alpha_{1}\right)\right)$, which is everywhere below that of the non-target group $\psi\left(\alpha_{2}\right)=1-G\left(\underline{\sigma}_{2}-\underline{\sigma}_{1}+G^{-1}\left(1-\alpha_{1}\right)\right)$. As a result, in a robust sorting equilibrium, target-group students will gamble up and apply to college 1 more often, and insure with an application to college 2 less often, than non-target group students.

Regarding common values, Theorem 5 (b) (proof in the online appendix) asserts that as the preference for a target group at college 1 rises from the no preference case $\pi_{1}=\pi_{2}=0$, it favors those target students, but now college 2 instead penalizes them. But when the preference for target students at the worse college 2 rises, both favor them.

In other words, an asymmetry emerges under common values. When only college 1 favors a target group of students, college 2 must counter this with a penalty, owing to two effects. First, the best favored students that previously applied to college 2 now just apply to college 1 , and thus the pool of target applicants at college 2 worsens. This portfolio effect was present with private values. Second, college 2 confronts an acceptance curse. A student who enrolls there either only applied to it, or also applied to college 1 - and was rejected. So the event that a student enrolls at college 2 is a worse signal of his caliber if college 1 has favored them.

\footnotetext{
${ }^{32}$ This asymmetric result should speak to studies, like Kane (1998), that found that affirmative action for disadvantaged minorities is generally confined to selective schools — as we think of college 1 .
} 


\section{Concluding Remarks}

We have formulated the college admissions problem for two ranked colleges with fixed capacities in order to study the effects of two frictions in equilibrium. Student types are heterogeneous and colleges only partially observe their types. College applications are costly, and students therefore face a nontrivial portfolio choice. This model admits a tractable separable solution in stages - student portfolios reflect the admission standards, and colleges then compete as if they were Bertrand duopolists. This framework is the only equilibrium model that speaks to the recent empirical explorations of student application behavior (Avery and Kane (2004); Pallais (2009); Carrell and Sacerdote (2012); Hoxby and Avery (2012)).

We have characterized in a testable fashion how student admission chances co-move as their calibers improve, showing how their optimal portfolio choices over stretch and safety schools differ. We have have discovered that even in this highly monotone matching world, sorting of students and colleges fails absent stronger assumptions. For better students need not always apply more aggressively: If the worse college is either too good or too small, or the application process is noisy enough, one student may gamble on the better college while a more talented one does not. Likewise, college admissions standards need not reflect their quality - the worse college may optimally impose higher standards if it is small enough. Large public schools might well be punished in college rankings publications that use SAT scores of enrolled students in ranking schools 33

Turning to affirmative action, we predict that favored minority applicants apply as ambitiously as if they were majority applicants of higher caliber. Card and Krueger (2005) investigate what happened when affirmative action was eliminated at state schools in California and Texas. They find a small but statistically significant drop in the probability that minority applicants send their SAT scores to elite state schools, but find no such effect for highly qualified minority applicants (those with high SATs or GPAs). This is consistent with our finding that lower caliber minority applicants send stretch applications under affirmative action.

While the two college world is restrictive, it is the most parsimonious model with portfolio effects, stretch and safety schools, and admission standards set by competing

\footnotetext{
33 Avery, Glickman, Hoxby, and Metrick (2004) develop an innovative revealed preference college ranking based on enrollment decisions by students admitted at multiple schools, and public schools are indeed ranked higher under their approach. One difficulty for them is that the initial application portfolios are endogenous. Explicitly modeling the application decision using our model of portfolio choice — as in $\mathrm{Fu}(2010)$ — may be helpful in resolving the econometric inference problems that result.
} 
schools. The portfolio effects induce important and realistic interdependencies missing from all frictionless models of student-college matching. Since assortative matching can fail in this setting, it can fail more generally. Our baseline model has assumed that students perfectly know their calibers and colleges only observe them with noise, but we have argued that the portfolio effects and sorting failures monotonically diminish as we shift toward the opposite extreme when colleges have superior information. And even in this limit, students send stretch and safety applications, and sorting can fail.

\section{A Appendix: Omitted Proofs}

\section{A.1 Colleges Optimally Employ Admissions Thresholds}

Let $\chi_{i}(\sigma)$ be the expected value of the student's caliber given that he applies to college $i$, his signal is $\sigma$, and he accepts. College $i$ optimally employs a threshold rule if, and only if, $\chi_{i}(\sigma)$ increases in $\sigma$. For college 1 this is immediate, since $g(\sigma \mid x)$ enjoys the MLRP property. College 2 faces an acceptance curse, and so $\chi_{2}(\sigma)$ is:

$$
\chi_{2}(\sigma)=\frac{\int_{\mathcal{C}_{2}} x g(\sigma \mid x) f(x) d x+\int_{\mathcal{B}} x G\left(\underline{\sigma}_{1} \mid x\right) g(\sigma \mid x) f(x) d x}{\int_{\mathcal{C}_{2}} g(\sigma \mid x) f(x) d x+\int_{\mathcal{B}} G\left(\underline{\sigma}_{1} \mid x\right) g(\sigma \mid x) f(x) d x}
$$

where we denote by $\mathcal{C}_{2}$ the set of calibers applying to 2 only, and $\mathcal{B}$ those applying to both ${ }^{34}$ Write $(11)$ as $\chi_{2}(\sigma)=\int_{\mathcal{B}_{\mathcal{C}}} x h_{2}(x \mid \sigma) d x$ using indicator function notation:

$$
h_{2}(x \mid \sigma)=\frac{\left(I_{\mathcal{C}_{2}}(x)+I_{\mathcal{B}} G\left(\underline{\sigma}_{1} \mid x\right)\right) g(\sigma \mid x) f(x)}{\int_{\mathcal{B} \cup \mathcal{C}_{2}}\left(I_{\mathcal{C}_{2}}(t)+I_{\mathcal{B}} G\left(\underline{\sigma}_{1} \mid t\right)\right) g(\sigma \mid t) f(t) d t},
$$

Then the 'density' $h_{2}(x \mid \sigma)$ has the MLRP. Therefore, $\chi_{2}(\sigma)$ increases in $\sigma$.

Notice that the same results obtain for any increasing function $\chi_{i}(\sigma)$ - in particular, if it is the identity function, as in the private values case.

\section{A.2 Simultaneous versus Sequential Timing}

We claim the subgame perfect equilibrium (SPE) outcomes of the two-stage game when students move first coincide with the Nash equilibria of the simultaneous-move game.

\footnotetext{
34 We assume that students employ pure strategies, which follows from our analysis of the student optimization in $\$ 3.1$. Measurability of sets $\mathcal{B}$ and $\mathcal{C}_{2}$ owe to the continuity of our functions $\alpha_{i}(x)$ in $\$ 3.2$
} 
First, consider an SPE of the two-stage game where students choose applications $S=$ $S(\cdot)$ and then colleges choose standards $\underline{\sigma}_{1}(S)$ and $\underline{\sigma}_{2}(S)$. Colleges best respond to each other given the realized $S$. As students are non atomic, they treat $S$ as fixed in the firststage, and so their applications best respond to the anticipated standards $\underline{\sigma}_{1}^{*}=\underline{\sigma}_{1}(S)$ and $\underline{\sigma}_{2}^{*}=\underline{\sigma}_{2}(S)$. This is a Nash equilibrium of the simultaneous move game.

Conversely, since each student has measure zero, he cannot affect the college standards by adjusting his application strategy. Hence, any equilibrium $\left(S, \underline{\sigma}_{1}^{*}, \underline{\sigma}_{2}^{*}\right)$ of the simultaneous move game is also an SPE outcome of the two-stage game.

\section{A.3 Acceptance Function Shape: Proof of Theorem 1}

To avoid duplication, we assume $\underline{\sigma}_{1}>\underline{\sigma}_{2}$ throughout the proof.

$(\Rightarrow)$ The Acceptance Function has the Double Secant Property. First, since $G\left(\underline{\sigma}_{1} \mid x\right)$ is continuously differentiable in $x$, the acceptance function is continuously differentiable on $(0,1]$. Given $\alpha \equiv 1-G(\sigma \mid \xi(\alpha, \sigma))$, partial derivatives have positive slopes $\xi_{\alpha}, \xi_{\sigma}>0$. Differentiating (3),

$$
\begin{array}{rrr}
\frac{\partial \psi}{\partial \alpha_{1}}= & -G_{x}\left(\underline{\sigma}_{2} \mid \xi\left(\alpha_{1}, \underline{\sigma}_{1}\right)\right) \xi_{\alpha}\left(\alpha_{1}, \underline{\sigma}_{1}\right)>0 \\
\frac{\partial \psi}{\partial \underline{\sigma}_{1}}= & -G_{x}\left(\underline{\sigma}_{2} \mid \xi\left(\alpha_{1}, \underline{\sigma}_{1}\right)\right) \xi_{\sigma}\left(\alpha_{1}, \underline{\sigma}_{1}\right)>0 \\
\frac{\partial \psi}{\partial \underline{\sigma}_{2}}= & -g\left(\underline{\sigma}_{2} \mid \xi\left(\alpha_{1}, \underline{\sigma}_{1}\right)\right)<0 .
\end{array}
$$

Properties of the cdf $G$ imply $\psi\left(0, \underline{\sigma}_{1}, \underline{\sigma}_{2}\right) \geq 0$ and $\psi\left(1, \underline{\sigma}_{1}, \underline{\sigma}_{2}\right)=1$. The limits of $\psi$ as thresholds approach the supremum and infimum owe to limit properties of $G$.

Now, $G(\sigma \mid x)$ and $1-G(\sigma \mid x)$ are strictly log-supermodular in $(\sigma, x)$ since the density $g(\sigma \mid x)$ obeys the strict MLRP. Since $x=\xi\left(\alpha_{1}, \underline{\sigma}_{1}\right)$ is strictly increasing in $\alpha_{1}$, $G\left(s \mid \xi\left(\alpha_{1}, \underline{\sigma}_{1}\right)\right)$ and $1-G\left(s \mid \xi\left(\alpha_{1}, \underline{\sigma}_{1}\right)\right)$ are then strictly log-supermodular in $\left(s, \alpha_{1}\right)$. So the secant slopes below strictly fall in $\alpha_{1}$, since $\underline{\sigma}_{1}>\underline{\sigma}_{2}$ :

$$
\frac{\psi\left(\alpha_{1}\right)}{\alpha_{1}}=\frac{1-G\left(\underline{\sigma}_{2} \mid \xi\left(\alpha_{1}\right)\right)}{1-G\left(\underline{\sigma}_{1} \mid \xi\left(\alpha_{1}\right)\right)} \quad \text { and } \quad \frac{1-\psi\left(\alpha_{1}\right)}{1-\alpha_{1}}=\frac{G\left(\underline{\sigma}_{2} \mid \xi\left(\alpha_{1}\right)\right)}{G\left(\underline{\sigma}_{1} \mid \xi\left(\alpha_{1}\right)\right)} .
$$

$(\Leftarrow)$ Deriving a Signal Distribution. Conversely, fix a function $h$ with the double secant property and a smoothly monotone onto function $\alpha_{1}(x)$. Also, put $\alpha_{2}(x)=$ $h\left(\alpha_{1}(x)\right)$, so that $\alpha_{2}(x)>\alpha_{1}(x)$. We must find a continuous signal density $g(\sigma \mid x)$ with the strict MLRP and thresholds $\sigma_{1}>\sigma_{2}$ that rationalize the $h$ as the acceptance function consistent with these thresholds and signal distribution. 
Step 1: A Discrete Signal Distribution. Consider a discrete distribution with realizations in $\{-1,0,1\}: g_{1}(x)=\alpha_{1}(x), g_{0}(x)=\alpha_{2}(x)-\alpha_{1}(x)$ and $g_{-1}(x)=1-\alpha_{2}(x)$. Indeed, for each caliber $x, g_{i} \geq 0$ and sum to 1 . This obeys the strict MLRP because

$$
\frac{g_{0}(x)}{g_{1}(x)}=\frac{\alpha_{2}(x)-\alpha_{1}(x)}{\alpha_{1}(x)}=\frac{h\left(\alpha_{1}(x)\right)}{\alpha_{1}(x)}-1
$$

is strictly decreasing by the first secant property of $h$, and

$$
\frac{g_{0}(x)}{g_{-1}(x)}=\frac{\alpha_{2}(x)-\alpha_{1}(x)}{1-\alpha_{2}(x)}=-1+\frac{1-\alpha_{1}(x)}{1-h\left(\alpha_{1}(x)\right)}
$$

is strictly increasing in $x$ by the second secant property of $h$.

Let the college thresholds be $\left(\underline{\sigma}_{1}, \underline{\sigma}_{2}\right)=(0.5,-0.5)$. Then $G\left(\underline{\sigma}_{1} \mid x\right)=g_{-1}(x)+$ $g_{0}(x)=1-\alpha_{1}(x)$ and $G\left(\underline{\sigma}_{2} \mid x\right)=g_{-1}(x)=1-\alpha_{2}(x)$. Rearranging yields $\alpha_{1}(x)=$ $1-G\left(\underline{\sigma}_{1} \mid x\right)$ and $\alpha_{2}(x)=1-G\left(\underline{\sigma}_{2} \mid x\right)$. Inverting $\alpha_{1}(x)$ and recalling that $\alpha_{2}=h\left(\alpha_{1}\right)$, we obtain $\alpha_{2}=h\left(\alpha_{1}\right)=1-G\left(\underline{\sigma}_{2} \mid \xi\left(\underline{\sigma}_{1}, \alpha_{1}\right)\right)$, thereby showing that $h$ is the acceptance function consistent with this signal distribution and thresholds.

Step 2: A Continuous Signal Density. To create an atomless signal distribution, we smooth the atoms using a carefully chosen kernel function. Define $g(\sigma \mid x)=$ $\sum_{i=\{-1,0,1\}} g_{i}(x) k(x, \sigma-i)$ and let $k(x, s)=1(|s|<0.5)(1+2 s w(x))$, where the weighting function $w(x)$ has range $[0,1]$. The transformation is mass-preserving since as $w(x)$ transfers mass to a point $s>0$ it removes the corresponding mass from $-s$. The weighting function determines the shape of the smoothing, so we now find conditions on $w(x)$ such that the strict MLRP holds. Consider $\sigma_{0}<\sigma_{1} \in(-1.5,1.5)$, and suppose first that they are both close to the same atom $i$ in that $\left|\sigma_{j}-i\right|<0.5$ for $j=0,1$. Then

$$
\frac{g\left(\sigma_{1} \mid x\right)}{g\left(\sigma_{0} \mid x\right)}=\frac{g_{i}(x)\left(1+2\left(\sigma_{1}-i\right) w(x)\right)}{g_{i}(x)\left(1+2\left(\sigma_{0}-i\right) w(x)\right)}=\frac{1+2\left(\sigma_{1}-i\right) w(x)}{1+2\left(\sigma_{0}-i\right) w(x)}
$$

which will be strictly increasing in $x$ if $w(x)$ is a strictly increasing function. Imposing this restriction on $w(x)$, if they inherit mass from points $i<j$, we have

$$
\frac{g\left(\sigma_{1} \mid x\right)}{g\left(\sigma_{0} \mid x\right)}=\frac{g_{k}(x)\left(1+2\left(\sigma_{1}-k\right) w(x)\right)}{g_{i}(x)\left(1+2\left(\sigma_{0}-i\right) w(x)\right)}
$$

A sufficient condition for this to be strictly increasing in $x$ almost everywhere is that it is weakly increasing when $\sigma_{1}-k=-0.5$ and $\sigma_{0}-i=0.5$ (i.e. $\frac{g_{k}(x)}{g_{i}(x)} \frac{(1-w(x))}{(1+w(x))}$ increasing 
$\forall k>i \forall x)$. Since $\frac{g_{k}(x)}{g_{i}(x)}$ is strictly increasing, choosing a strictly increasing $w(x)$ with appropriately bounded derivatives achieves this.

\section{A.4 Monotone Student Strategies}

Claim 1 Student behavior is monotone in caliber if (a) college 2 has payoff $u \leq 0.5$, and (b) college 2 imposes a low enough admissions standard relative to college 1.

By part $(a)$, if a student applies to college 1, then any better student does too. By part $(b)$, if a student applies to college 2 , then any worse student applies there or nowhere.

The proof proceeds as follows. We first show that $(i) u \leq 0.5$ implies that if a caliber applies to college 1 , then any higher caliber applies as well. Second, we (ii) produce a sufficient condition that ensures that the admissions threshold at college 2 is sufficiently lower than that of college 1 , so that if a caliber applies to college 2, then any lower caliber who applies to college sends an application to college 2, and calibers at the lower tail apply nowhere. From these two results, monotone student behavior ensues.

Proof of Part $(i)$, Step 1. We first show that the acceptance function $\alpha_{2}=\psi\left(\alpha_{1}\right)$ crosses $\alpha_{2}=(1 / u)\left(1-c / \alpha_{1}\right)$ (i.e., $\left.M B_{12} \equiv \alpha_{1}\left(1-\alpha_{2} u\right)=c\right)$ only once when $u \leq 0.5$. Since $(i)$ the acceptance function starts at $\alpha_{1}=0$ and ends at $\alpha_{1}=1$, (ii) $M B_{12}=c$ starts at $\alpha_{1}=c$ and ends at $\alpha_{1}=c /(1-u)$, and $(i i i)$ both functions are continuous, there exists a crossing point. And they intersect when $\alpha_{1}\left(1-\psi\left(\alpha_{1}\right) u\right)=c$.

$\left[\left(1-\psi\left(\alpha_{1}\right) u\right) \alpha_{1}\right]^{\prime}=1-u \psi\left(\alpha_{1}\right)-\alpha_{1} u \psi^{\prime}\left(\alpha_{1}\right)>1-u \psi\left(\alpha_{1}\right)-u \psi\left(\alpha_{1}\right)=1-2 u \psi\left(\alpha_{1}\right) \geq 1-2 u \geq 0$

where the first inequality exploits $\psi\left(\alpha_{1}\right) / \alpha_{1}$ falling in $\alpha_{1}$ (Theorem 1), i.e. $\psi^{\prime}\left(\alpha_{1}\right)<$ $\psi\left(\alpha_{1}\right) / \alpha_{1}$; the next two inequalities use $\psi\left(\alpha_{1}\right) \leq 1$ and $u \leq 0.5$. Since $M B_{12}$ is rising in $\alpha_{1}$ when the acceptance relation hits $\alpha_{2}=\left(1-c / \alpha_{1}\right) / u$, the intersection is unique.

Proof of Part $(i)$, Step 2. We now show that Step 1 implies the following single crossing property in terms of $x$ : if caliber $x$ applies to college 1 (i.e., if $1 \in S(x)$, then any caliber $y>x$ also applies to college 1 (i.e., $1 \in S(y)$ ). Suppose not; i.e., assume that either $S(y)=\varnothing$ or $S(y)=\{2\}$. If $S(y)=\varnothing$, then $S(x)=\varnothing$ as well, as $\alpha_{1}(x)<\alpha_{1}(y)$ and $\alpha_{2}(x)<\alpha_{2}(y)$, contradicting the hypothesis that $1 \in S(x)$. If $S(y)=\{2\}$, then there are two cases: $S(x)=\{1\}$ or $S(x)=\{1,2\}$. The first cannot occur, for by Theorem $11 \alpha_{2}(x) / \alpha_{1}(x)>\alpha_{2}(y) / \alpha_{1}(y)$, and thus $\alpha_{2}(y) u \geq \alpha_{1}(y)$ implies $\alpha_{2}(x) u>\alpha_{1}(x)$, contradicting $S(x)=\{1\}$. In turn, the second case is ruled out by the 
monotonicity of $M B_{12}$ derived above, as caliber $y$ has greater incentives than $x$ to add college 1 to its portfolio, and thus $S(y)=\{2\}$ cannot be optimal.

Proof of Part (ii), Step 1. We first show that if the acceptance function passes above the point $\left(\bar{\alpha}_{1}, \bar{\alpha}_{2}\right)=(u(1-\sqrt{1-4 c / u}) / 2,(1-\sqrt{1-4 c / u}) / 2)$ - point $P$ in the right panel of Figure 6 - then there is a unique crossing of the acceptance function and $\alpha_{2}=c / u\left(1-\alpha_{1}\right)$, i.e. $M B_{21}=c$. Now, the acceptance function passes above $\left(\bar{\alpha}_{1}, \bar{\alpha}_{2}\right)$ if

$$
\psi\left(\bar{\alpha}_{1}, \underline{\sigma}_{1}, \underline{\sigma}_{2}\right) \geq \bar{\alpha}_{2} .
$$

This condition relates $\underline{\sigma}_{1}$ and $\underline{\sigma}_{2}$. Rewrite (14) using Theorem 1 as $\underline{\sigma}_{2} \leq \eta\left(\underline{\sigma}_{1}\right)<\underline{\sigma}_{1}$, where $\eta$ is defined by $\psi\left(\bar{\alpha}_{1}, \underline{\sigma}_{1}, \eta\right)=\bar{\alpha}_{2}$. Thus, condition (14) requires a large enough "wedge" between the standards of the two colleges.

To show that (14) implies a unique crossing, consider the secant of $\alpha_{2}=c / u\left(1-\alpha_{1}\right)$ (the curve $M B_{21}=c$ ). It has an increasing secant if and only if $\alpha_{1} \geq 1 / 2$. To see this, differentiate $\alpha_{2} / \alpha_{1}=c / u \alpha_{1}\left(1-\alpha_{1}\right)$ in $\alpha_{1}$. Notice also that $M B_{21}=c$ intersects the diagonal $\alpha_{2}=\alpha_{1}$ at the points $\left(\alpha_{1}^{\ell}, \alpha_{2}^{\ell}\right)=(1 / 2-\sqrt{1-4 c / u} / 2,1 / 2-\sqrt{1-4 c / u} / 2)$ and $\left(\alpha_{1}^{h}, \alpha_{2}^{h}\right)=(1 / 2+\sqrt{1-4 c / u} / 2,1 / 2+\sqrt{1-4 c / u} / 2)>(1 / 2,1 / 2)$.

Condition (14) gives $\psi\left(\alpha_{1}^{\ell}, \underline{\sigma}_{1}, \underline{\sigma}_{2}\right)>\alpha_{2}^{\ell}$. Since $\underline{\sigma}_{2}<\underline{\sigma}_{1}$, we have $\psi\left(\alpha_{1}, \underline{\sigma}_{1}, \underline{\sigma}_{2}\right) \geq \alpha_{2}$ for all $\alpha_{1} \geq \bar{\alpha}_{1}$. Thus, the acceptance function crosses $M B_{21}=c$ at or above $\left(\alpha_{1}^{h}, \alpha_{2}^{h}\right)$. And since $\alpha_{1}^{h}>1 / 2$, the secant of $M B_{21}=c$ must be increasing at any intersection with the acceptance function. Hence, there must be a single crossing point.

Proof of PART (ii), Step 2. We now show that this single crossing property in $\alpha$ implies another in $x$ : If caliber $x$ applies to college 2 (i.e., if $2 \in S(x)$ ), then any caliber $y<x$ that applies somewhere also applies to college 2 (i.e., $2 \in S(y)$ if $S(y) \neq \varnothing)$. Suppose not; i.e., assume that $S(y)=\{1\}$. Then there are two cases: $S(x)=\{2\}$ or $S(x)=\{1,2\}$. The first cannot occur, for by Theorem $1 \alpha_{2}(x) / \alpha_{1}(x)<\alpha_{2}(y) / \alpha_{1}(y)$, and thus $\alpha_{2}(x) u \geq \alpha_{1}(x)$ implies $\alpha_{2}(y) u>\alpha_{1}(y)$, contradicting $S(x)=\{2\}$. The second case is ruled out by the monotonicity of $M B_{21}$ given condition (14), as caliber $y$ has greater incentives than $x$ to apply to college 2 , and thus $S(y)=\{1\}$ cannot be optimal. Finally, $S(y)=\varnothing$ if $\alpha_{2}(y) u<c$ by (14), which happens for low calibers below a threshold.

\section{A.5 The Law of Demand}

Claim 2 (The Falling Demand Curve) If either college raises its admission standard, then its enrollment falls, and thus its rival's enrollment rises. 
We only consider the case when $\underline{\sigma}_{1}$ rises, since the argument for $\underline{\sigma}_{2}$ is similar. Also, we focus on the nontrivial portfolio effect in each case, since the standards effect of an increase in $\underline{\sigma}_{1}$ is immediate: it lowers enrollment in college 1, and raises it in college 2 .

Proof Step 1: The applicant pool at College 1 Shrinks. When $\underline{\sigma}_{1}$ rises, the acceptance relation shifts up by Claim 1, and thus the above type sets change as well. Fix a caliber $x \in \mathcal{C}_{2}$ or $x \in \Phi$, so that $1 \notin S(x)$ We will show that $x$ continues to apply either to college 2 only or nowhere, and thus the pool of applicants at college 1 shrinks because $\alpha_{1}(x)$ declines. If $x \in \mathcal{C}_{2}$, then $\alpha_{2}(x) u-c \geq 0$ and $\alpha_{2}(x) u \geq \alpha_{1}(x)$, and this continues to hold after the increase in $\underline{\sigma}_{1}$, since $\alpha_{1}(x)$ falls while $\alpha_{2}(x)$ is constant. And if $x \in \Phi$, then clearly caliber $x$ will continue to apply nowhere when $\underline{\sigma}_{1}$ increases.

Proof Step 2: The applicant pool at College 2 expands. It suffices to show that if any caliber $x$ that applies to college 2 at $\underline{\sigma}_{1}$ also applies there at a higher $\underline{\sigma}_{1}$. Fix a caliber $x \in \mathcal{C}_{2}$ or $x \in \mathcal{B}$, so that $2 \in S(x)$. If $x \in \mathcal{C}_{2}$, then $\alpha_{2}(x) u-c \geq 0$ and $\alpha_{2}(x) u \geq \alpha_{1}(x)$; these inequalities continue to hold after $\underline{\sigma}_{1}$ rises, since $\alpha_{1}(x)$ falls while $\alpha_{2}(x)$ remains constant. And if $x \in \mathcal{B}$, then $M B_{21}=\left(1-\alpha_{1}(x)\right) \alpha_{2}(x) u$ rises in $\underline{\sigma}_{1}$, encouraging caliber $x$ to apply to college 2 . So $x \notin \mathcal{C}_{1} \cup \Phi$ even after $\underline{\sigma}_{1}$ rises.

\section{A.6 Existence: Proof of Theorem 2}

For definiteness, we now denote the infimum (supremum) signal by $-\infty(\infty)$.

DeFinition of $\bar{\kappa}_{1}\left(\kappa_{2}, c\right)$. We will choose the capacity $\bar{\kappa}_{1}$ given $\kappa_{2}$ so that when college 2 has no standards, both colleges exactly fill their capacity. This borderline capacity is less than $1-\kappa_{2}$ since a positive mass of students - perversely, those with the highest calibers - applies just to college 1, and some are rejected.

Fix any $\kappa_{2} \in(0,1)$, and let $\underline{\sigma}_{1}^{L}\left(\kappa_{2}\right)$ be the unique solution to $\kappa_{2}=\mathcal{E}_{2}\left(\underline{\sigma}_{1},-\infty\right)$, i.e., when college 2 accepts everybody. (Existence and uniqueness of $\underline{\sigma}_{1}^{L}\left(\kappa_{2}\right)$ follows from $\mathcal{E}_{2}(-\infty,-\infty)=0, \mathcal{E}_{2}(\infty,-\infty)=1$, and $\mathcal{E}_{2}\left(\underline{\sigma}_{1},-\infty\right)$ increasing and continuous in $\left.\underline{\sigma}_{1}.\right)$

Define the threshold capacity $\bar{\kappa}_{1}\left(\kappa_{2}\right)=\mathcal{E}_{1}\left(\underline{\sigma}_{1}^{L}\left(\kappa_{2}\right),-\infty\right)$.

Limiting BeHavior of $\bar{\kappa}_{1}\left(\kappa_{2}, c\right)$. Since $\kappa_{2}=\mathcal{E}_{2}\left(\underline{\sigma}_{1}^{L}\left(\kappa_{2}\right),-\infty\right), \bar{\kappa}_{1}\left(\kappa_{2}\right)$ equals $1-\kappa_{2}$ minus the mass of students who only applied to, and were rejected by, college 1 . This mass vanishes as $c$ vanishes, for then everybody applies to both colleges. Therefore, the threshold $\bar{\kappa}_{1}\left(\kappa_{2}\right)$ converges to $1-\kappa_{2}$ as $c$ goes to zero.

\footnotetext{
${ }^{35}$ With a slight abuse of notation, we let $\Phi$ denote the set of calibers that apply nowhere. The same symbol was previously used to denote the analogous set in $\alpha$-space.
} 


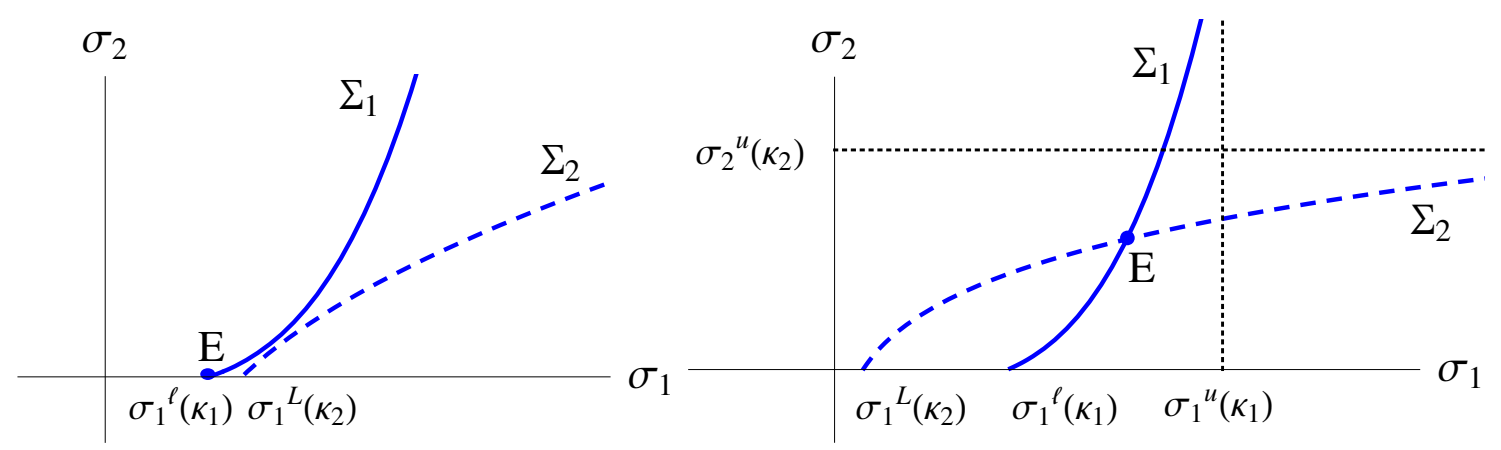

Figure 8: Equilibrium Existence. In the left panel, since $\kappa_{1}>\bar{\kappa}_{1}\left(\kappa_{2}\right)$, the best response functions $\Sigma_{1}$ and $\Sigma_{2}$ do not intersect, and equilibrium is at $E$ with $\underline{\sigma}_{2}=0$. The right panel depicts the proof of Claim 2 for the case $\kappa_{1}<\bar{\kappa}_{1}\left(\kappa_{2}\right)$.

Robust Stable Equilibrium with Excess Capacity. Let $\kappa_{1} \geq \bar{\kappa}_{1}\left(\kappa_{2}\right)$. We claim that there exists a robust stable equilibrium in which college 2 accepts everybody, and college 1 sets a threshold $\underline{\sigma}_{1}^{\ell}\left(\kappa_{1}\right)$, the unique solution to $\kappa_{1}=\mathcal{E}_{1}\left(\underline{\sigma}_{1},-\infty\right)$, which satisfies $\underline{\sigma}_{1}^{\ell}\left(\kappa_{1}\right) \leq \underline{\sigma}_{1}^{L}\left(\kappa_{2}\right)$. For since college 2 rejects no one, $\underline{\sigma}_{1}^{\ell}\left(\kappa_{1}\right)$ fills college 1 's capacity exactly. The enrollment at college 2 is then $\mathcal{E}_{2}\left(\underline{\sigma}_{1}^{\ell}\left(\kappa_{1}\right),-\infty\right) \leq \kappa_{2}\left(\right.$ as $\underline{\sigma}_{1}^{\ell}\left(\kappa_{1}\right) \leq$ $\underline{\sigma}_{1}^{L}\left(\kappa_{2}\right)$ and $\mathcal{E}_{2}\left(\underline{\sigma}_{1}, \underline{\sigma}_{2}\right)$ is increasing in $\left.\underline{\sigma}_{1}\right)$, so by accepting everybody college 2 fills as much capacity as it can. This robust equilibrium is trivially stable, as $\Sigma_{2}$ is 'flat' at the crossing point (see Figure 8, left panel). Moreover, if $\kappa_{1}>\bar{\kappa}_{1}\left(\kappa_{2}\right)$, then college 2 has excess capacity in this equilibrium.

Robust Stable Equilibrium without Excess Capacity. Assume now $\kappa_{1}<$ $\bar{\kappa}_{1}\left(\kappa_{2}\right)$. We will show that the continuous functions $\Sigma_{1}$ and $\Sigma_{2}$ must cross at least once (i.e., a robust equilibrium exists), and that the slope condition is met (i.e., it is stable). First, in this case $\underline{\sigma}_{1}^{L}\left(\kappa_{2}\right)<\underline{\sigma}_{1}^{\ell}\left(\kappa_{1}\right)$ or, equivalently, $\Sigma_{2}^{-1}\left(-\infty, \kappa_{2}\right)<\Sigma_{1}\left(-\infty, \kappa_{1}\right)$. Second, as the standard of college 2 goes to infinity, college 1's threshold converges to $\underline{\sigma}_{1}^{u}\left(\kappa_{1}\right)<\infty$, the unique solution to $\kappa_{1}=\mathcal{E}_{1}\left(\underline{\sigma}_{1}, \infty\right)$. This is the largest threshold that college 1 can set given $\kappa_{1}$. Similarly, as the standard of college 1 goes to infinity, college 2's threshold converges to $\underline{\sigma}_{2}^{u}\left(\kappa_{2}\right)<\infty$, the unique solution to $\kappa_{2}=\mathcal{E}_{2}\left(\infty, \underline{\sigma}_{2}\right)$, i.e. the largest threshold that college 2 can set given $\kappa_{2}$. Third, $\Sigma_{1}$ and $\Sigma_{2}$ are continuous. By the Intermediate Value Theorem, they must cross at least once with the slope condition being satisfied (see Figure 8, right panel). Thus, a robust stable equilibrium exists when $\kappa_{1}<\bar{\kappa}_{1}\left(\kappa_{2}\right)$. Moreover, in any robust equilibrium there is no excess capacity at either college, since the best response functions satisfy $\Sigma_{2}^{-1}\left(-\infty, \kappa_{2}\right)<\Sigma_{1}\left(-\infty, \kappa_{1}\right)$.

Hence, a robust stable equilibrium exists for any $\kappa_{2} \in(0,1)$. Capacities are filled 
when $\kappa_{1} \leq \bar{\kappa}_{1}\left(\kappa_{2}\right)$, while there can be excess capacity at college 2 if $\kappa_{1}>\bar{\kappa}_{1}\left(\kappa_{2}\right)$.

\section{A.7 Stochastic Dominance in Robust Sorting Equilibria}

Claim 3 (Sorting and the Caliber Distribution) In any robust sorting equilibrium, the caliber distribution at college 1 first-order stochastically dominates that at college 2.

Proof Step 1: Monotone Student Strategy Representation. A monotone student strategy is represented by the partition of the set of types:

$$
\Phi=\left[0, \xi_{2}\right), \mathcal{C}_{2}=\left[\xi_{2}, \xi_{B}\right), \mathcal{B}=\left[\xi_{B}, \xi_{1}\right), \mathcal{C}_{1}=\left[\xi_{1}, \infty\right)
$$

where $\xi_{2}<\xi_{B}<\xi_{1}$ are defined by the intersection of the acceptance function with $c / u$, $\alpha_{2}=\left(1-c / \alpha_{1}\right) / u\left(\right.$ i.e., $\left.M B_{12}=c\right)$, and $\alpha_{2}=c /\left[u\left(1-\alpha_{1}\right)\right]$ (i.e., $\left.M B_{21}=c\right)$, respectively.

Proof Step 2: Enrolled Caliber Densities. Fix $\underline{\sigma}_{1}$ and $\underline{\sigma}_{2}$. Let $f_{1}(x)$ and $f_{2}(x)$ be the densities of calibers enrolled at colleges 1 and 2, respectively. Formally,

$$
\begin{aligned}
f_{1}(x) & =\frac{\alpha_{1}(x) f(x)}{\int_{\xi_{B}}^{\infty} \alpha_{1}(t) f(t) d t} I_{\left[\xi_{B}, \infty\right)}(x) \\
f_{2}(x) & =\frac{I_{\left[\xi_{2}, \xi_{B}\right]}(x) \alpha_{2}(x) f(x)+\left(1-I_{\left[\xi_{2}, \xi_{B}\right]}(x)\right) \alpha_{2}(x)\left(1-\alpha_{1}(x)\right) f(x)}{\int_{\xi_{2}}^{\xi_{B}} \alpha_{2}(s) f(s) d s+\int_{\xi_{B}}^{\xi_{1}} \alpha_{2}(s)\left(1-\alpha_{1}(s)\right) f(s) d s} I_{\left[\xi_{2}, \xi_{1}\right]}(x),
\end{aligned}
$$

where $I_{A}$ is the indicator function of the set $A$.

Proof Step 3: Log-Supermodularity of $f_{i}(x)$. We shall show that, if $x_{L}, x_{H} \in$ $[0, \infty)$, with $x_{H}>x_{L}$, then $f_{1}\left(x_{H}\right) f_{2}\left(x_{L}\right) \geq f_{2}\left(x_{H}\right) f_{1}\left(x_{L}\right)$; i.e., $f_{i}(x)$ is log-supermodular in $(-i, x)$, or it satisfies MLRP. The result follows as MLRP implies that the cdfs are ordered by first-order stochastic dominance.

Using (16) and (17), $f_{1}\left(x_{H}\right) f_{2}\left(x_{L}\right) \geq f_{2}\left(x_{H}\right) f_{1}\left(x_{L}\right)$ is equivalent to

$$
\begin{aligned}
& \alpha_{1 H} I_{\left[\xi_{B}, \infty\right)}\left(x_{H}\right)\left(I_{\left[\xi_{2}, \xi_{B}\right]}\left(x_{L}\right) \alpha_{2 L}+\left(1-I_{\left[\xi_{2}, \xi_{B}\right]}\left(x_{L}\right)\right) \alpha_{2 L}\left(1-\alpha_{1 L}\right)\right) I_{\left[\xi_{2}, \xi_{1}\right]}\left(x_{L}\right) \geq \\
& \alpha_{1 L} I_{\left[\xi_{B}, \infty\right)}\left(x_{L}\right)\left(I_{\left[\xi_{2}, \xi_{B}\right]}\left(x_{H}\right) \alpha_{2 H}+\left(1-I_{\left[\xi_{2}, \xi_{B}\right]}\left(x_{H}\right)\right) \alpha_{2 H}\left(1-\alpha_{1 H}\right)\right) I_{\left[\xi_{2}, \xi_{1}\right]}\left(x_{H}\right),
\end{aligned}
$$

where $\alpha_{i j}=\alpha_{i}\left(x_{j}\right), i=1,2, j=L, H$. It is easy to show that the only nontrivial case is when $x_{L}, x_{H} \in\left[\xi_{B}, \xi_{1}\right]$ (in all the other cases, either both sides are zero, or only the right 
side is). If $x_{L}, x_{H} \in\left[\xi_{B}, \xi_{1}\right]$, then 18$)$ becomes $\alpha_{1 H} \alpha_{2 L}\left(1-\alpha_{1 L}\right) \geq \alpha_{1 L} \alpha_{2 H}\left(1-\alpha_{1 H}\right)$, or

$$
\begin{aligned}
& \left(1-G\left(\underline{\sigma}_{1} \mid x_{H}\right)\right)\left(1-G\left(\underline{\sigma}_{2} \mid x_{L}\right)\right) G\left(\underline{\sigma}_{1} \mid x_{L}\right) \geq \\
& \left(1-G\left(\underline{\sigma}_{1} \mid x_{L}\right)\right)\left(1-G\left(\underline{\sigma}_{2} \mid x_{H}\right)\right) G\left(\underline{\sigma}_{1} \mid x_{H}\right) .
\end{aligned}
$$

Since $g(\sigma \mid x)$ satisfies MLRP, it follows that $G(\sigma \mid x)$ is decreasing in $x$, and hence $G\left(\underline{\sigma}_{1} \mid x_{L}\right) \geq G\left(\underline{\sigma}_{1} \mid x_{H}\right)$. Next, $1-G(\sigma \mid x)$ is log-supermodular in $(x, \sigma)$, and hence

$$
\left(1-G\left(\underline{\sigma}_{1} \mid x_{H}\right)\right)\left(1-G\left(\underline{\sigma}_{2} \mid x_{L}\right)\right) \geq\left(1-G\left(\underline{\sigma}_{1} \mid x_{L}\right)\right)\left(1-G\left(\underline{\sigma}_{2} \mid x_{H}\right)\right),
$$

as $\underline{\sigma}_{1}>\underline{\sigma}_{2}$ in a robust sorting equilibrium. Thus, (19) holds, thereby proving that $f_{i}(x)$ is log-supermodular in $(-i, x)$, and so $F_{1}$ first-order stochastically dominates $F_{2}$.

\section{A.8 Comparative Statics: Proof of Theorem 3}

(a) An Increase in College 1's Payoff. Let the initial equilibrium point $E_{0}$ be $\left(\underline{\sigma}_{1}^{e}, \underline{\sigma}_{2}^{e}\right)$, and let the best reply loci after the increase in $v$ be $\Sigma_{1}^{\prime}\left(\sigma_{2}\right)$ and $\Sigma_{2}^{\prime}\left(\sigma_{1}\right)$. Consider $\underline{\sigma}_{2}^{\prime}=\Sigma_{1}^{\prime-1}\left(\underline{\sigma}_{1}^{e}\right)$ (i.e. it is the $\underline{\sigma}_{2}$ at which college 1's standards remain unchanged as $v$ increases). Since $v$ increasing unambiguously shifts $\Sigma_{1}$ to the right, $\underline{\sigma}_{2}^{\prime}<\underline{\sigma}_{2}^{e}$.

Now consider the point $A=\left(\underline{\sigma}_{1}^{e}, \underline{\sigma}_{2}^{\prime}\right)$ shown in figure 5 , and assume that student applications are monotone at $A$ (see the online appendix for the non-monotone case). Then let the thresholds at $E_{0}$ be $\left(\xi_{2}^{e}, \xi_{B}^{e}, \xi_{1}^{e}\right)$ and at $A$ be $\left(\xi_{2}^{\prime}, \xi_{B}^{\prime}, \xi_{1}^{\prime}\right)$. Since college 2 has lower standards, $\xi_{2}^{\prime}<\xi_{2}^{e}$. Recall that the marginal benefit of an insurance application is $\left(1-\alpha_{1}\right) \alpha_{2} u$. Since standards at college 1 are the same as before and the standards at college 2 are lower, the payoff to insurance is higher and $\xi_{1}^{\prime}>\xi_{1}^{e}$. So the set of applicants to college 2 is strictly bigger than before. Also since college 1's standards and capacity at $A$ remain the same, its application set $\left[\xi_{B}, \infty\right)$ must be the same as before, so that $\xi_{B}^{e}=\xi_{B}^{\prime}$. Since $\underline{\sigma}_{2}^{\prime}<\underline{\sigma}_{2}^{e}$, enrollment must rise on $\left[\xi_{2}^{e}, \xi_{B}^{e}\right)$ and $\left[\xi_{B}^{e}, \xi_{1}^{e}\right]$, and so even ignoring the new applicants, college 2 has excess demand at $A$.

Thus, $\Sigma_{2}^{\prime}\left(\underline{\sigma}_{1}^{e}\right)>\sigma_{2}^{\prime}$, which implies that $\Sigma_{2}^{\prime}$ lies above the point $A$, and therefore above $\Sigma_{1}^{\prime}$ at that point (by construction of $A$ ). Since $\Sigma_{1}^{\prime}$ is eventually entirely above $\Sigma_{2}^{\prime}$ (see the proof of Theorem 2), they must cross somewhere to the right of $A$, and so there exists a robust stable equilibrium $E_{1}$ in which $\underline{\sigma}_{1}>\underline{\sigma}_{1}^{e}$.

(b) An InCREAse In THE COMMON COST $c$. To align the logic with (A), we prove instead that a decrease in $c$ increases $\underline{\sigma}_{1}$. Then $\Sigma_{1}$ shifts right but $\Sigma_{2}$ shifts ambiguously. The result follows as in (A) if there is excess demand at $A$. Relative to $E_{0}$, at $A$ 
applications are cheaper and standards at college 2 have fallen. So again $\xi_{2}^{\prime}<\xi_{2}^{e}$ and $\xi_{1}^{\prime}>\xi_{1}^{e}$. By the argument in $(\mathrm{A}), \xi_{B}^{e}=\xi_{B}^{\prime}$, so enrollment rises on $\left[\xi_{2}^{e}, \xi_{B}^{e}\right)$ and $\left[\xi_{B}^{e}, \xi_{1}^{e}\right]$, yielding the result.

(c) InCREASES IN INDIVIDUAL COSTS. We modify 15 for different costs: $\xi_{1}$ is defined by $M B_{21}=c_{2}$, $\xi_{B}$ by $M B_{12}=c_{1}$, and $\xi_{2}$ by $\alpha_{2} u=c_{2}$. If $c_{2}$ rises, then $\xi_{1}$ drops, $\xi_{2}$ rises, and $\xi_{B}$ is unchanged; thus, the applicant pool at college 2 shrinks, and at college 1 is unchanged. So the $\Sigma_{2}$ curve shifts down, while $\Sigma_{1}$ remains unchanged. The functions cross at a lower threshold pair, and so both standards $\underline{\sigma}_{1}, \underline{\sigma}_{2}$ both fall. Next consider an increase in $c_{1}$. This raises $\xi_{B}$, which shrinks the applicant pool at college 1 , and increases the enrollment at college 2 , at a fixed admission standard. This shifts $\Sigma_{1}$ left and $\Sigma_{2}$ up. While the effect on the standard $\underline{\sigma}_{2}$ is ambiguous, we now deduce that $\underline{\sigma}_{1}$ falls. Differentiating (6) with respect to $c_{1}$, and using Cramer's Rule:

$$
\frac{\partial \underline{\sigma}_{1}}{\partial c_{1}}=\frac{\left(\partial \mathcal{E}_{2} / \partial c_{1}\right)\left(\partial \mathcal{E}_{1} / \partial \underline{\sigma}_{2}\right)-\left(\partial \mathcal{E}_{1} / \partial c_{1}\right)\left(\partial \mathcal{E}_{2} / \partial \underline{\sigma}_{2}\right)}{\left(d \mathcal{E}_{1} / d \underline{\sigma}_{1}\right)\left(d \mathcal{E}_{2} / d \underline{\sigma}_{2}\right)-\left(d \mathcal{E}_{2} / d \underline{\sigma}_{1}\right)\left(d \mathcal{E}_{1} / d \underline{\sigma}_{2}\right)}
$$

Since the robust equilibrium is stable, the slope of $\Sigma_{1}$ is steeper that of $\Sigma_{2}$, and thus the denominator is positive. Let $P_{i}(\xi \mid y)$ be the portfolio density shift to college $i$ at type $\xi$ given an increment to standard or cost $y$, and let $S_{2}(A)$ be the own-standards effect at college 2 in set $A$. Then parse the enrollment derivatives into the portfolio and standards effects: $d \mathcal{E}_{1} / d c_{1}=P_{1}\left(\xi_{B} \mid c_{1}\right)<0, d \mathcal{E}_{2} / d \underline{\sigma}_{2}=\Sigma_{i=2, B, 1} P_{2}\left(\xi_{i} \mid \underline{\sigma}_{2}\right)-S_{2}\left(C_{2}\right)-S_{2}(B)<0$, $d \mathcal{E}_{2} / d c_{1}=P_{2}\left(\xi_{B} \mid c_{1}\right)>0$, and $d \mathcal{E}_{1} / d \underline{\sigma}_{2}=P_{1}\left(\xi_{B} \mid \underline{\sigma}_{2}\right)>0$. If $c_{1}$ slightly rises, then $\xi_{B}$ rises by some $\delta>0$. Thus, college 1 loses mass $f\left(\xi_{B}\right) \alpha_{1} \delta$ of students, and college 2 gains mass $f\left(\xi_{B}\right) \alpha_{1} \alpha_{2} \delta$ of students who would have gone to college 1 . Likewise, if $\underline{\sigma}_{2}$ slightly rises, then $\xi_{B}$ falls by some $\delta^{\prime}$, and college 1 gains mass $f\left(\xi_{B}\right) \alpha_{1} \delta^{\prime}$ and college 2 loses mass $f\left(\xi_{B}\right) \alpha_{1} \alpha_{2} \delta^{\prime}$. Thus, $P_{1}\left(\xi_{B} \mid \underline{\sigma}_{2}\right) P_{2}\left(\xi_{B} \mid c_{1}\right)-P_{1}\left(\xi_{B} \mid c_{1}\right) P_{2}\left(\xi_{B} \mid \underline{\sigma}_{2}\right)$ equals

$$
\left[f\left(\xi_{B}\right) \alpha_{1} \delta^{\prime}\right]\left[f\left(\xi_{B}\right) \alpha_{1} \alpha_{2} \delta\right]-\left[f\left(\xi_{B}\right) \alpha_{1} \delta\right]\left[f\left(\xi_{B}\right) \alpha_{1} \alpha_{2} \delta^{\prime}\right]=0
$$

Hence, the numerator in 20 reduces to

$$
-P_{1}\left(\xi_{B} \mid c_{1}\right)\left[P_{2}\left(\xi_{2} \mid \underline{\sigma}_{2}\right)+P\left(\xi_{1} \mid \underline{\sigma}_{2}\right)-S_{2}\left(\mathcal{C}_{2}\right)-S_{2}(\mathcal{B})\right]<0
$$

(d) Caliber improvement. In a robust sorting equilibrium, the applicant pool at college 1 consists of calibers $x \in\left[\xi_{B}, \infty\right)$. When $\underline{\sigma}_{1}$ falls in equilibrium, $\xi_{B}$ must rise since college 1 has the same capacity as before. Let $\left(\xi_{B}^{0}, \underline{\sigma}_{1}^{0}\right)$ be the old equilibrium pair 
and $\left(\xi_{B}^{1}, \underline{\sigma}_{1}^{1}\right)$ the new one, with $\xi_{B}^{0}<\xi_{B}^{1}$ and $\underline{\sigma}_{1}^{0}>\underline{\sigma}_{1}^{1}$. Then the distribution function of enrolled students at college 1 under equilibrium $i=0,1$ is:

$$
F_{1}^{i}(x)=\frac{\int_{\xi_{B}^{i}}^{x}\left(1-G\left(\underline{\sigma}_{1}^{i} \mid t\right)\right) f(t) d t}{\int_{\xi_{B}^{i}}^{\infty}\left(1-G\left(\underline{\sigma}_{1}^{i} \mid t\right)\right) f(t) d t}
$$

We must show $F_{1}^{1}(x) \leq F_{1}^{0}(x)$ for all $x \in\left[\xi_{B}^{1}, \infty\right)$. For any $x$, the denominators on both sides equal $k_{1}$, so cancel them. Now notice that $0=F_{1}^{1}\left(\xi_{B}^{1}\right)<F_{1}^{0}\left(\xi_{B}^{1}\right)$ and $\lim _{x \rightarrow \infty} F_{1}^{1}(x)=\lim _{x \rightarrow \infty} F_{1}^{0}(x)=1$. Since both functions are continuous in $x$, if $\partial F_{1}^{1} / \partial x>\partial F_{1}^{0} / \partial x$ for all $x \in\left[\xi_{B}^{1}, \infty\right)$, then $F_{1}^{1}(x)<F_{1}^{0}(x)$. But this requires $\left(1-G\left(\underline{\sigma}_{1}^{1} \mid x\right)\right) f(x)>\left(1-G\left(\underline{\sigma}_{1}^{0} \mid x\right)\right) f(x)$, which follows from $\underline{\sigma}_{1}^{1}<\underline{\sigma}_{1}^{0}$.

\section{A.9 Sorting and Non-Sorting Equilibria: Proof of Theorem 4}

Part (a): College 2 Is Too Good. We construct acceptance chances $\left(\alpha_{1}(x), h\left(\alpha_{1}(x)\right)\right)$ such that student behavior is non-monotone, college enrollment equals capacity, and $\alpha_{1}(x)$ and $h\left(\left(\alpha_{1}(x)\right)\right.$ obey the requirements of Theorem 1 . Then that theorem yields existence of a signal distribution with non-monotone equilibrium student behavior.

Step 1: The Acceptance Function And Student Behavior. When $u>0.5$, the secant from the origin to $M B_{12}=c$ falls as $\alpha_{1}$ tends to $c /(1-u)$ - as in the left panel of Figure 6. So for some $\underline{z}<c /(1-u)$, a line from the origin to $(\underline{z}, 1)$ slices the $M B_{12}$ curve twice. Let $h:[0,1] \rightarrow[0,1]$ be defined by $h\left(\alpha_{1}\right)=\alpha_{1} / \underline{z}$ and on $[0, \underline{z})$, and $h\left(\alpha_{1}\right)=1$ for $\alpha_{1} \geq \underline{z}$. Observe that $h(0)=0$ and $h(1)=1$, and that $h$ is weakly increasing, with both $h\left(\alpha_{1}\right) / \alpha_{1}$ and $\left[1-h\left(\alpha_{1}\right)\right] /\left[1-\alpha_{1}\right]$ weakly decreasing, so $h$ obeys the double secant property. Now consider student behavior. Moving along the acceptance relation $h$, students with acceptance chance $\alpha_{1}<(c \bar{z}) / u$ will apply nowhere; those in the interval $[(c \bar{z}) / u, \underline{a})$ will apply to college 2 only; those between $[\underline{a}, \bar{a}]$ will apply to both; those in $(\bar{a}, c /(1-u))$ will apply to college 2 only, those in $[c /(1-u), 1-(c / u)]$ will apply to both, and those above $1-(c / u)$ will apply to college 1 ; where $\underline{a}, \bar{a}$ are the pair of intersections with the $M B_{12}$ curve. Hence, student behavior is non-monotone.

Step 2: Transforming the type SPACE. Notice that if one chooses a monotone function $\alpha_{1}(x)$, then we induce a distribution $G(a)=\mathbb{P}\left(\alpha_{1}(X)<a\right)$ (i.e., the probability that a random student $X$ has acceptance chance at college 1 less than $a$ ). Conversely, we can obtain any distribution $G(a)$ by choosing $\alpha_{1}(x)=G^{-1}(F(x))$. The resulting function $\alpha_{1}(x)$ thus obtained will be smooth, monotone and onto provided that $G$ has a 

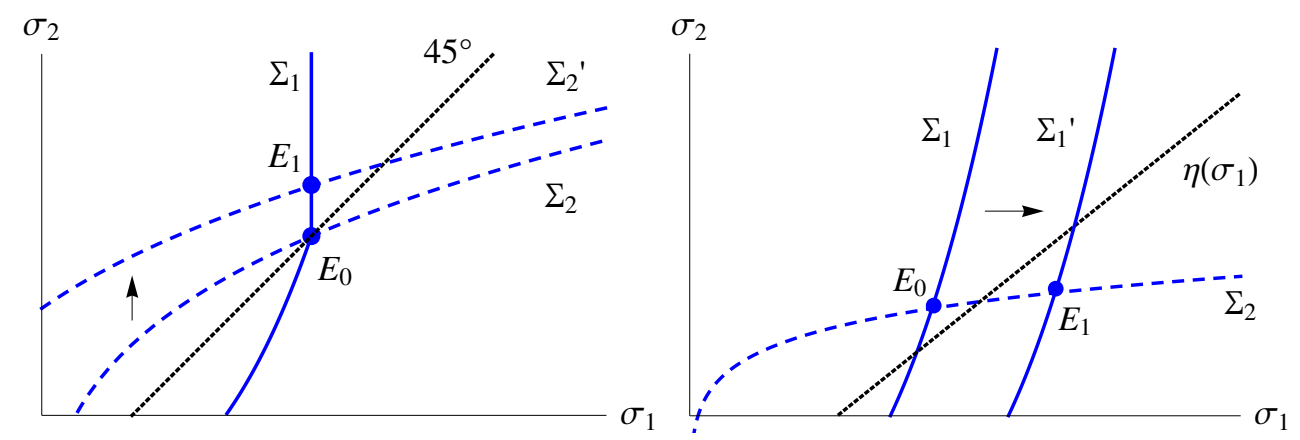

Figure 9: Existence of Sorting and Non-Sorting Robust Equilibria. We depict the proof of Theorem 4 . In the left panel, when $\kappa_{2}$ falls, $\Sigma_{2}$ shifts up to $\Sigma_{2}^{\prime}$, inducing a robust non-sorting equilibrium at $E_{1}$. In the right panel, when $\kappa_{1}$ falls, $\Sigma_{1}$ shifts right to $\Sigma_{1}^{\prime}$. The standards at the new robust equilibrium $E_{1}$ obey $\underline{\sigma}_{2}<\eta\left(\underline{\sigma}_{1}\right)$, i.e., the equilibrium is sorting.

continuous and strictly positive density over $[0,1]$. Therefore, we can restate our problem as choosing a $G$ with these properties and such that the enrollment equations hold.

Step 3: Enrollment. The enrollment equations are given by:

$$
\begin{aligned}
& \int_{\underline{a}}^{\bar{a}} a d G(a)+\int_{\frac{c}{1-u}}^{1} a d G(a)=\kappa_{1} \\
& \int_{\frac{c \bar{z}}{u}}^{\underline{a}} \frac{a}{\bar{z}} d G(a)+\int_{\underline{a}}^{\bar{a}}(1-a) \frac{a}{\bar{z}} d G(a)+\int_{\bar{a}}^{\bar{z}} \frac{a}{\bar{z}} d G(a)+\int_{\bar{z}}^{\frac{c}{1-u}} d G(a)+\int_{\frac{c}{1-u}}^{1-\frac{c}{u}}(1-a) d G(a)=\kappa_{2} .
\end{aligned}
$$

We now decouple the equations by letting $G(\bar{a})-G(\underline{a})=G(1-(c / u))-G(c /(1-u))=$ $\epsilon$ for some small $\epsilon$ (e.g. by using a uniform density). It is clear that the resulting individual integral equations have an infinite number of solutions for $G(a)$ on those regions. Choosing one of them completes the proof. For then Theorem 1 yields a signal density $g(\sigma \mid x)$ and thresholds $\underline{\sigma}_{1}>\underline{\sigma}_{2}$ such that $h\left(\alpha_{1}(x)\right)$ is the acceptance function.

Part $(b)$ : College 2 is Too Small. The proof is constructive. Consider $\left(\alpha_{1}, \alpha_{2}\right)=(c, c / u)$ on the line $\alpha_{2}=\alpha_{1} / u$. The acceptance function evaluated at $\alpha_{1}=c$ lies below $c / u$ when

$$
\psi\left(c, \underline{\sigma}_{1}, \underline{\sigma}_{2}\right)<c / u .
$$

We will restrict attention to pairs $\left(\underline{\sigma}_{1}, \underline{\sigma}_{2}\right)$ such that 21$)$ holds. In this case, any student who applies to college starts by adding college 1 to his portfolio, and this happens as 
soon as $\alpha_{1}(x) \geq c$, or when $x \geq \xi\left(c, \underline{\sigma}_{1}\right)$. Then enrollment at college 1 is given by

$$
\mathcal{E}_{1}\left(\underline{\sigma}_{1}, \underline{\sigma}_{2}\right)=\int_{\xi\left(c, \underline{\sigma}_{1}\right)}^{\infty}\left(1-G\left(\underline{\sigma}_{1} \mid x\right)\right) f(x) d x
$$

which is independent of $\underline{\sigma}_{2}$. Thus, for any capacity $\kappa_{1} \in(0,1)$, a unique threshold $\underline{\sigma}_{1}\left(\kappa_{1}\right)$ solves $\kappa_{1}=\mathcal{E}_{1}\left(\underline{\sigma}_{1}, \underline{\sigma}_{2}\right)$. (The $\Sigma_{1}^{-1}$ function is "vertical" when 21 holds, since the applicant pool at college 1 does not depend on college 2's admissions threshold.)

The analysis above allows us to restrict attention to finding robust equilibria within the set of $\left(\underline{\sigma}_{1}, \underline{\sigma}_{2}\right)$ such that $\underline{\sigma}_{1}=\underline{\sigma}_{1}\left(\kappa_{1}\right)$ and $\underline{\sigma}_{2}$ satisfies $\psi\left(c, \underline{\sigma}_{1}\left(\kappa_{1}\right), \underline{\sigma}_{2}\right)<c / u$.

Enrollment at college 2 is given by

$$
\mathcal{E}_{2}\left(\underline{\sigma}_{1}\left(\kappa_{1}\right), \underline{\sigma}_{2}\right)=\int_{B} G\left(\underline{\sigma}_{1}\left(\kappa_{1}\right) \mid x\right)\left(1-G\left(\underline{\sigma}_{2} \mid x\right)\right) f(x) d x
$$

which is continuous, decreasing in $\underline{\sigma}_{2}$, and increasing in $\underline{\sigma}_{1}$ (see Claim 2). Thus, $\kappa_{2}=$ $\mathcal{E}_{2}\left(\underline{\sigma}_{1}\left(\kappa_{1}\right), \underline{\sigma}_{2}\right)$ yields $\underline{\sigma}_{2}=\Sigma_{2}\left(\underline{\sigma}_{1}\left(\kappa_{1}\right), \kappa_{2}\right)$, which is strictly decreasing in $\kappa_{2}$.

Given $\kappa_{1}$, let $\bar{\kappa}_{2}\left(\kappa_{1}\right)=\mathcal{E}_{2}\left(\underline{\sigma}_{1}\left(\kappa_{1}\right), \underline{\sigma}_{1}\left(\kappa_{1}\right)\right)$ be such that equilibrium ensues if both colleges set the same threshold ${ }^{36}$ Since $\Sigma_{2}$ strictly falls in $\kappa_{2}$, for any $\kappa_{2}<\bar{\kappa}_{2}\left(\kappa_{1}\right)$, a robust equilibrium exists with $\underline{\sigma}_{2}>\underline{\sigma}_{1}\left(\kappa_{1}\right)$. Then $(i)$ for any $\kappa_{1} \in(0,1)$ and $\kappa_{2} \in$ $\left(0, \bar{\kappa}_{2}\left(\kappa_{1}\right)\right]$, there is a unique robust equilibrium with $\underline{\sigma}_{1}=\underline{\sigma}_{1}\left(\kappa_{1}\right)$ and $\underline{\sigma}_{2} \geq \underline{\sigma}_{1}\left(\kappa_{1}\right)$, having (ii) non-monotone college and student behavior (Figure 9, left).37

Part $(c)$ : Conditions for Equilibrium Sorting. We prove that there exists $\underline{\kappa}_{1}\left(\kappa_{2}\right)>0$ such that if $\kappa_{1} \leq \underline{\kappa}_{1}\left(\kappa_{2}\right)$ and $u \leq 0.5$, then there are only robust sorting equilibria and neither college has excess capacity.

Fix $\kappa_{2} \in(0,1)$. We first show that the robust stable equilibrium with no excess capacity derived in Claim 2 is also sorting when the capacity of college 1 is small enough. More precisely, there is a threshold $\underline{\kappa}_{1}\left(\kappa_{2}\right)$, smaller than the bound $\bar{\kappa}_{1}\left(\kappa_{2}\right)$ defined in the proof of Claim 2, such that for all $\kappa_{1} \in\left(0, \underline{\kappa}_{1}\left(\kappa_{2}\right)\right)$, there is a pair of admissions thresholds $\left(\underline{\sigma}_{1}, \underline{\sigma}_{2}\right)$ that satisfies $\kappa_{1}=\mathcal{E}_{1}\left(\underline{\sigma}_{1}, \underline{\sigma}_{2}\right), \kappa_{2}=\mathcal{E}_{2}\left(\underline{\sigma}_{1}, \underline{\sigma}_{2}\right)$, and $\underline{\sigma}_{2}<\eta\left(\underline{\sigma}_{1}\right)$ (i.e., a robust sorting equilibrium), and $\partial \Sigma_{1} / \partial \underline{\sigma}_{2} \partial \Sigma_{2} / \partial \underline{\sigma}_{1}<1$ (i.e., the robust equilibrium is stable).

The proof uses three easily-verified properties of the $\eta$ function implicitly defined by inequality (14): (a) $\eta$ is strictly increasing; (b) $\underline{\sigma}_{2}=\eta\left(\underline{\sigma}_{1}\right) \rightarrow \infty$ as $\underline{\sigma}_{1} \rightarrow \infty ;(c)$ $\underline{\sigma}_{1}=\eta^{-1}\left(\underline{\sigma}_{2}\right) \rightarrow-\infty$ as $\underline{\sigma}_{2} \rightarrow-\infty$.

\footnotetext{
${ }^{36}$ It is not difficult to show that $\psi\left(c, \underline{\sigma}_{1}, \underline{\sigma}_{2}\right)<c / u$ is satisfied if $\underline{\sigma}_{2} \geq \underline{\sigma}_{1}\left(\kappa_{1}\right)$.

${ }^{37} \mathrm{We}$ are not ruling out the existence of another robust equilibrium that does not satisfy 21 .
} 
For any $\kappa_{1} \in\left(0, \bar{\kappa}_{1}\left(\kappa_{2}\right)\right)$, we know from Claim 2 that there exists a pair $\left(\underline{\sigma}_{1}, \underline{\sigma}_{2}\right)$ that satisfies $\kappa_{1}=\mathcal{E}_{1}\left(\underline{\sigma}_{1}, \underline{\sigma}_{2}\right)$ and $\kappa_{2}=\mathcal{E}_{2}\left(\underline{\sigma}_{1}, \underline{\sigma}_{2}\right)$, with $\left(\partial \Sigma_{1} / \partial \underline{\sigma}_{2}\right)\left(\partial \Sigma_{2} / \partial \underline{\sigma}_{1}\right)<1$.

Claim 4 The pair $\left(\underline{\sigma}_{1}, \underline{\sigma}_{2}\right)$ is a robust sorting equilibrium when $\kappa_{1}$ is sufficiently small.

Proof: Let $M\left(\kappa_{2}\right)=\left\{\left(\underline{\sigma}_{1}, \underline{\sigma}_{2}\right) \mid \kappa_{2}=\mathcal{E}_{2}\left(\underline{\sigma}_{1}, \underline{\sigma}_{2}\right)\right.$ and $\left.\underline{\sigma}_{2}=\eta\left(\underline{\sigma}_{1}\right)\right\}$. Graphically, this is the set of all pairs at which $\underline{\sigma}_{2}=\Sigma_{2}\left(\underline{\sigma}_{1}, \kappa_{2}\right)$ crosses $\underline{\sigma}_{2}=\eta\left(\underline{\sigma}_{1}\right)$.

If $M\left(\kappa_{2}\right)=\varnothing$ we are done, for then $\underline{\sigma}_{2}=\Sigma_{2}\left(\underline{\sigma}_{1}, \kappa_{2}\right)<\eta\left(\underline{\sigma}_{1}\right)$ for all $\underline{\sigma}_{1}$, including those at which $\kappa_{1}=\mathcal{E}_{1}\left(\underline{\sigma}_{1}, \underline{\sigma}_{2}\right)$ and $\kappa_{2}=\mathcal{E}_{2}\left(\underline{\sigma}_{1}, \underline{\sigma}_{2}\right)$. To see this, note that $(i) \underline{\sigma}_{1}=$ $\eta^{-1}\left(\underline{\sigma}_{2}\right) \rightarrow-\infty$ as $\underline{\sigma}_{2} \rightarrow-\infty$, while we proved in Claim 2 that $\underline{\sigma}_{1}=\Sigma_{2}^{-1}\left(\underline{\sigma}_{2}, \kappa_{2}\right)$ converges to $\underline{\sigma}_{1}^{l}\left(\kappa_{2}\right)>-\infty$. Also, $($ ii $) \underline{\sigma}_{2}=\eta\left(\underline{\sigma}_{1}\right) \rightarrow \infty$ as $\underline{\sigma}_{1} \rightarrow \infty$, while we proved in Claim 2 that $\underline{\sigma}_{2}=\Sigma_{2}\left(\underline{\sigma}_{1}, \kappa_{2}\right)$ converges to $\underline{\sigma}_{2}^{u}\left(\kappa_{2}\right)<\infty$. Properties $(i)$ and $(i i)$ reveal that if $\Sigma_{2}$ and $\eta$ do not intersect, then $\Sigma_{2}$ is everywhere below $\eta$.

If $M\left(\kappa_{2}\right) \neq \varnothing$, let $\left(\underline{\sigma}_{1}^{s}\left(\kappa_{2}\right), \underline{\sigma}_{2}^{s}\left(\kappa_{2}\right)\right)=\sup M\left(\kappa_{2}\right)$, which is finite by property $(b)$ of $\eta\left(\underline{\sigma}_{1}\right)$ and since $\underline{\sigma}_{2}=\Sigma_{2}\left(\underline{\sigma}_{1}, \kappa_{2}\right)$ converges to $\underline{\sigma}_{2}^{u}\left(\kappa_{2}\right)<\infty$ as $\underline{\sigma}_{1}$ goes to infinity (see the proof of Claim 2). Now, as $\kappa_{1}$ goes to zero, $\underline{\sigma}_{1}=\Sigma_{1}\left(\underline{\sigma}_{2}, \kappa_{1}\right)$ goes to infinity for any value of $\underline{\sigma}_{2}$, for college 1 becomes increasingly more selective to fill its dwindling capacity. Since $\underline{\sigma}_{2}$ is bounded above by $\underline{\sigma}_{2}^{u}\left(\kappa_{2}\right)$, there exists a threshold $\underline{\kappa}_{1}\left(\kappa_{2}\right) \leq \bar{\kappa}_{1}\left(\kappa_{2}\right)$ such that, for all $\kappa_{1} \in\left(0, \underline{\kappa}_{1}\left(\kappa_{2}\right)\right)$, the aforementioned pair $\left(\underline{\sigma}_{1}, \underline{\sigma}_{2}\right)$ that satisfies $\kappa_{1}=\mathcal{E}_{1}\left(\underline{\sigma}_{1}, \underline{\sigma}_{2}\right)$ and $\kappa_{2}=\mathcal{E}_{2}\left(\underline{\sigma}_{1}, \underline{\sigma}_{2}\right)$ is strictly bigger than $\left(\underline{\sigma}_{1}^{s}\left(\kappa_{2}\right), \underline{\sigma}_{2}^{s}\left(\kappa_{2}\right)\right)$, thereby showing that it also satisfies $\underline{\sigma}_{2}<\eta\left(\underline{\sigma}_{1}\right)$. Hence, a robust sorting stable equilibrium exists for any $\kappa_{2}$ and $\kappa_{1} \in\left(0, \underline{\kappa}_{1}\left(\kappa_{2}\right)\right)$, with both colleges filling their capacities (see Figure 9, right panel).

To finish the proof, notice that, if there are multiple robust equilibria, both colleges fill their capacity in all of them (graphically, the conditions on capacities ensure that $\Sigma_{2}$ starts above $\Sigma_{1}$ for low values of $\underline{\sigma}_{1}$ and eventually ends below it). Moreover, adjusting the bound $\underline{\kappa}_{1}\left(\kappa_{2}\right)$ downward if needed, all robust equilibria are sorting (graphically, for $\kappa_{1}$ sufficiently small, the set of pairs at which $\Sigma_{1}$ and $\Sigma_{2}$ intersect are all below $\eta$ ).

\section{A.10 Affiliated Evaluations}

We now show that if students and colleges see noisy conditionally iid signals of calibers, then this is formally a special case of $(\star)$.

Let $t$ be a student's true caliber, unknown to him and colleges. It has density $p(t)$ on $[0,1]$. After seeing the signal realization $X=x$, drawn with type-dependent density $f(x \mid t)$, the student updates his beliefs to $p(t \mid x)=f(x \mid t) p(t) / f(x)$. If a student of caliber 
$t$ applies to a college, the college observes a signal $\sigma$ drawn with density $\gamma(\sigma \mid t)$ and cdf $\Gamma(\sigma \mid t)$ on $[0,1]$. If a student applies to both colleges, then they observe conditionally iid signals. We assume that $f(x \mid t)$ and $\gamma(\sigma \mid t)$ obey the strict MLRP.

Define the conditional joint density of signals $g\left(\sigma_{1}, \sigma_{2} \mid x\right)=\int_{0}^{1} \gamma\left(\sigma_{1} \mid t\right) \gamma\left(\sigma_{2} \mid t\right) p(t \mid x) d t$. Notice that $g$ integrates to 1 , and so is a valid density. Also, as an integral of products of log-supermodular functions, it inherits this property, by Karlin and Rinott $(1980)$. In other words, the signals are affiliated. Next, define the density $f(x)=\int_{0}^{1} f(x \mid t) p(t) d t$. We now reinterpret the signal $x$ in the conditional iid case as the student true caliber. To show that this model is a special case of $(\star)$, we prove that student and college optimizations have the same solutions as in the conditional iid case.

Student BeHAvior. It suffices to express the chances of two acceptance events for the conditional iid model without reference to the type $t$, and thus as in the affiliated model $(\star)$. First, the unconditional acceptance chance at college $i=1,2$ is

$\alpha_{i}(x)=\int_{0}^{1}\left(1-\Gamma\left(\underline{\sigma}_{i} \mid t\right)\right) p(t \mid x) d t=\int_{0}^{1} \int_{\underline{\sigma}_{i}}^{1} \gamma\left(\sigma_{i} \mid t\right) p(t \mid x) d \sigma_{i} d t=\int_{\underline{\sigma}_{i}}^{1} \int_{0}^{1} g\left(\sigma_{i}, \sigma_{j} \mid x\right) d \sigma_{j} d \sigma_{i}$

Next, the probability of being rejected at 1 and accepted at 2 is

$$
\begin{aligned}
\int_{0}^{1} \Gamma\left(\underline{\sigma}_{1} \mid t\right)\left(1-\Gamma\left(\underline{\sigma}_{2} \mid t\right)\right) p(t \mid x) d t & =\int_{0}^{1} \int_{0}^{\underline{\sigma}_{1}} \int_{\underline{\sigma}_{2}}^{1} \gamma\left(\sigma_{2} \mid t\right) \gamma\left(\sigma_{1} \mid t\right) p(t \mid x) \sigma_{1} d \sigma_{2} d t \\
& =\int_{0}^{\underline{\sigma}_{1}} \int_{\underline{\sigma}_{2}}^{1} g\left(\sigma_{1}, \sigma_{2} \mid x\right) d \sigma_{1} d \sigma_{2} .
\end{aligned}
$$

College Behavior. It likewise suffices to express the enrollment functions without reference to the student type $t$. For instance, for college 1,

$$
\begin{aligned}
\mathcal{E}_{1}\left(\underline{\sigma}_{1}, \underline{\sigma}_{2}\right) & =\int_{0}^{1}\left(\int_{\mathcal{C}_{1} \cup \mathcal{B}} f(x \mid t) d x\right)\left(\int_{\underline{\sigma}_{1}}^{1} \gamma\left(\sigma_{1} \mid t\right) d \sigma_{1}\right) p(t) d t \\
& =\int_{\mathcal{C}_{1} \cup \mathcal{B}} \int_{\underline{\sigma}_{1}}^{1}\left(\int_{0}^{1} \gamma\left(\sigma_{1} \mid t\right) f(x \mid t) p(t) d t\right) d \sigma_{1} d x \\
& =\int_{\mathcal{C}_{1} \cup \mathcal{B}}\left(\int_{\underline{\sigma}_{1}}^{1} \int_{0}^{1} g\left(\sigma_{1}, \sigma_{2} \mid x\right) d \sigma_{2} d \sigma_{1}\right) f(x) d x
\end{aligned}
$$

The analysis of college 2 is analogous and thus omitted. 


\section{A.11 Affirmative Action: Proof of Theorem 5 (a)}

Step 1: Equilibrium Conditions. Here, we assume a continuous signal cdf derivative $G_{x}$. Given any discount pair $\left(\Delta_{1}, \Delta_{2}\right)$, the capacity equations with two groups are:

$$
\begin{aligned}
& \kappa_{1}=\phi \mathcal{E}_{1}^{\tau}\left(\underline{\sigma}_{1}-\Delta_{1}, \underline{\sigma}_{2}-\Delta_{2}\right)+(1-\phi) \mathcal{E}_{1}^{N}\left(\underline{\sigma}_{1}, \underline{\sigma}_{2}\right) \\
& \kappa_{2}=\phi \mathcal{E}_{2}^{\tau}\left(\underline{\sigma}_{1}-\Delta_{1}, \underline{\sigma}_{2}-\Delta_{2}\right)+(1-\phi) \mathcal{E}_{2}^{N}\left(\underline{\sigma}_{1}, \underline{\sigma}_{2}\right),
\end{aligned}
$$

where $\mathcal{E}_{i}^{\tau}, \mathcal{E}_{i}^{N}$ are the respective fractions of targeted and non-targeted groups enrolled at college $i$, defined just as in (4) and (5), for the sets of signals (15). Since the signal density $g=G_{\sigma}$ and its derivative $G_{x}$ are both continuous, all derivatives of the enrollment function (using Leibnitz rule) are continuous too.

Step 2: Single College Preference Case. Differentiating equations 22 and (23) with respect to $\Delta_{1}$ :

$$
\begin{aligned}
\frac{J}{\phi} \frac{\partial \underline{\sigma}_{1}}{\partial \Delta_{1}} & =\sum_{i=1,2}(-1)^{i+1} \frac{\partial \mathcal{E}_{i}^{\tau}}{\partial\left(\underline{\sigma}_{1}-\Delta_{1}\right)}\left(\phi \frac{\partial \mathcal{E}_{3-i}^{\tau}}{\partial\left(\underline{\sigma}_{2}-\Delta_{2}\right)}+(1-\phi) \frac{\partial \mathcal{E}_{3-i}^{N}}{\partial \underline{\sigma}_{2}}\right) \\
\frac{J}{\phi} \frac{\partial \underline{\sigma}_{2}}{\partial \Delta_{1}} & =\sum_{i=1,2}(-1)^{i} \frac{\partial \mathcal{E}_{i}^{\tau}}{\partial\left(\underline{\sigma}_{1}-\Delta_{1}\right)}\left(\phi \frac{\partial \mathcal{E}_{3-i}^{\tau}}{\partial\left(\underline{\sigma}_{1}-\Delta_{1}\right)}+(1-\phi) \frac{\partial \mathcal{E}_{3-i}^{N}}{\partial \underline{\sigma}_{1}}\right)
\end{aligned}
$$

where the denominator, from Cramer's Rule, equals

$$
\begin{aligned}
J= & \left(\phi \frac{\partial \mathcal{E}_{1}^{\tau}}{\partial\left(\underline{\sigma}_{1}-\Delta_{1}\right)}+(1-\phi) \frac{\partial \mathcal{E}_{1}^{N}}{\partial \underline{\sigma}_{1}}\right)\left(\phi \frac{\partial \mathcal{E}_{2}^{\tau}}{\partial\left(\underline{\sigma}_{2}-\Delta_{2}\right)}+(1-\phi) \frac{\partial \mathcal{E}_{2}^{N}}{\partial \underline{\sigma}_{2}}\right) \\
& -\left(\phi \frac{\partial \mathcal{E}_{1}^{\tau}}{\partial\left(\underline{\sigma}_{2}-\Delta_{2}\right)}+(1-\phi) \frac{\partial \mathcal{E}_{1}^{N}}{\partial \underline{\sigma}_{2}}\right)\left(\phi \frac{\partial \mathcal{E}_{2}^{\tau}}{\partial\left(\underline{\sigma}_{1}-\Delta_{1}\right)}+(1-\phi) \frac{\partial \mathcal{E}_{2}^{N}}{\partial \underline{\sigma}_{1}}\right)
\end{aligned}
$$

is positive in any robust stable equilibrium - i.e. the two group version of the condition that the slope of $\Sigma_{1}$ exceed the slope of $\Sigma_{2}$ in $\$$ and $\$$ A.8. Now, $\partial \underline{\sigma}_{1} / \partial \Delta_{1}=\phi>0$ and $\partial \underline{\sigma}_{2} / \partial \Delta_{1}=0$ when $\Delta_{1}=\Delta_{2}=0$, because the derivatives of the function $\mathcal{E}_{i}^{\tau}$, $\mathcal{E}_{i}^{N}$ at colleges $i=1,2$ coincide. Thus, the feedback effects vanish when $\Delta_{1}=\Delta_{2}=0$, and are negligible in a neighborhood of it, by continuity of the enrollment derivatives.

Since $\Delta_{1}=\pi_{1}$, it follows that $\underline{\sigma}_{1}$ increases while $\underline{\sigma}_{1}-\Delta_{1}$ decreases when $\pi_{1}$ goes up, as $\partial\left(\underline{\sigma}_{1}-\Delta_{1}\right) /\left.\partial \pi_{1}\right|_{\pi_{1}=0}=\phi-1<0$.

The analysis of the derivatives of $\underline{\sigma}_{i}, i=1,2$, with respect to $\Delta_{2}$ is analogous.

Step 3: Both College Preference Case. Suppose now that $\pi_{1}=\pi_{2}=\pi=0$ 
and thus $\Delta_{1}=\Delta_{2}=\Delta=0$. Now let $\pi$ increase. Replacing in the analysis above $\Delta_{i}, i=1,2$, by $\Delta$ and differentiating, yields, after evaluating the expression at $\Delta=0$, $\partial \underline{\sigma}_{i} / \partial \Delta=\phi>0, i=1,2$. As before $\underline{\sigma}_{i}-\Delta$ goes down, thereby proving the result.

\section{References}

Albrecht, J., P. Gautier, and S. Vroman (2003): "Equilibrium Directed Search with Multiple Applications," Tinbergen Institute Discussion Papers.

Anderson, A., And L. Smith (2010): "Assortative Matching with Evolving Human Capital," Review of Economic Studies, 77(1), 3-29.

Avery, C., M. Glickman, C. Hoxby, And A. Metrick (2004): "A Revealed Preference Ranking of U.S. Colleges and Universities," NBER Working Paper 10830.

Avery, C., And T. Kane (2004): "Student Perceptions of College Opportunities: The Boston COACH Program," in College Choices: The Economics of Where to Go, When to Go, and How to Pay for It, ed. by C. Hoxby, pp. 355-393. University of Chicago Press.

Avery, C., ANd J. Levin (2010): "Early Admissions at Selective Colleges," American Economic Review, 100(5), 2125-2156.

Azevedo, E., And J. Leshno (2012): "A Supply and Demand Framework for TwoSided Matching Markets," Working paper, Harvard University.

Bettinger, E. P., B. T. Long, P. Oreopoulos, and L. Sanbonmatsu (2009): "The Role of Simplification and Information in College Decisions: Results from the H\&R Block FAFSA Experiment," NBER Working Paper 15361.

Burdett, K., And K. Judd (1983): "Equilibrium Price Dispersion," Econometrica, $51(4), 955-69$.

Burdett, K., S. Shi, and R. Wright (2001): "Pricing and Matching with Frictions," Journal of Political Economy, 109(5), 1060-1085.

Card, D., and A. B. Krueger (2005): "Would the Elimination of Affirmative Action Affect Highly Qualified Minority Applicants? Evidence from California and Texas," Industrial and Labor Relations Review, 58(3). 
Carrell, S., And B. Sacerdote (2012): "Late Interventions Matter Too: The Case of College Coaching in New Hampshire," Working Paper, Dartmouth College.

Chade, H. (2006): "Matching with noise and the acceptance curse," Journal of Economic Theory, 129(1), 81-113.

Chade, H., and L. Smith (2006): "Simultaneous Search," Econometrica, 74(5), 12931307.

Che, Y.-K., And Y. KoH (2013): "Decentralized College Admissions," Working Paper, Columbia University.

Curs, B., And L. Singell (2002): "An analysis of the application and enrollment processes for in-state and out-state students at a large public university," Economics of Education Review, 21, 111-124.

Epple, D., R. Romano, and H. Sieg (2006): "Admission, Tuition, and Financial Aid Policies in the Market for Higher Education," Econometrica, 74(4), 885-928.

- (2008): "Diversity and Affirmative Action in Higher Education," Journal of Public Economic Theory, 10(4), 81-113.

Fu, C. (2010): "Equilibrium Tuition, Applications, Admissions and Enrollment in the College Market," Working Paper, University of Wisconsin.

Gale, D., And L. Shapley (1962): "College Admissions and Stability of Marriage," American Mathematical Monthly, 69(1), 9-15.

Groen, J., And M. White (2004): "In-state versus out-state students: the divergence of interest between public universities and state governments," Journal of Public Economics, 88(9), 1793-1814.

Hickman, B. (2010): "Effort, Race Gap and Affirmative Action: A Game Theoretic Analysis of College Admissions," Working Paper, University of Chicago.

HoxBy, C. (2009): "The Changing Selectivity of American Colleges," Journal of Economic Perspectives, 23(4), 95-118.

Hoxby, C. M., And C. Avery (2012): "The Missing "One-Offs": The Hidden Supply of High-Achieving, Low Income Students," NBER Working Paper 18586. 
Kane, T. (1998): "Racial and ethnic preferences in college admissions," in The BlackWhite Test Score Gap, ed. by C. Jencks, and M. Phillips. Brookings Institution Press, Washington, D.C.

Karlin, S., And Y. RinotT (1980): "Classes of orderings of measures and related correlation inequalities. I. Multivariate totally positive distributions," Journal of Multivariate Analysis, 10, 467-498.

Kircher, P., and M. Galenianos (2006): "Directed Search with Multiple Job Applications," mimeo, University of Pennsylvania.

Pallais, A. (2009): "Small Differences that Matter: Mistakes in Applying to College," Working Paper, Harvard University.

Persico, N. (2000): "Information Acquisition in Auctions," Econometrica, 68(1), 135148.

Shimer, R., And L. Smith (2000): "Assortative Matching and Search," Econometrica, $68(2), 343-369$.

Smith, L. (2006): "Assortative Matching and Search," Journal of Political Economy, $114(6), 1124-1146$.

SteinberG, J. (2010): "Colleges Market Easy, No-Fee Sell to Applicants," New York Times. 